\title{
Silicon-based Anode for Lithium-ion Batteries: Effectiveness of Materials Synthesis and Electrode Preparation
}

\author{
Anix Casimir, ${ }^{\mathrm{a}}$ Hanguang Zhang, ${ }^{\mathrm{a}}$ Ogechi Ogoke ${ }^{\mathrm{a}}$, Joseph C. \\ Amine, ${ }^{\mathrm{b}}$ Jun $\mathrm{Lu},{ }^{\mathrm{c}, *}$ and Gang $\mathrm{Wu},{ }^{\mathrm{a}, *}$ \\ ${ }^{a}$ Department of Chemical and Biological Engineering, University at Buffalo, State University \\ of New York, Buffalo, NY 14260, United States. \\ ${ }^{\mathrm{b}}$ Material Science Division, Argonne National Laboratory, Argonne, IL, 60439, \\ United States. \\ ${ }^{\mathrm{c}}$ Chemical Sciences and Engineering Division, Argonne National Laboratory, \\ Argonne, IL, 60439, United States.
}

Review article submitted to Nano Energy

* Corresponding authors: gangwu@ buffalo.edu (G. Wu) and junlu@anl.gov (J. Lu) 
Abstract: Lithium-ion batteries are widely used throughout the world for portable electronic devices and mobile phones and show great potential for more demanding applications like electric vehicles. Unfortunately, lithium-ion batteries still lack the required level of energy storage to completely meet the demands of such applications as electric vehicles. Among advanced materials being studied, silicon nanoparticles have demonstrated great potential as an anode material to replace the commonly used graphite. Silicon has been shown to have a high theoretical gravimetric capacity, approximately $4200 \mathrm{mAh} / \mathrm{g}$, compared to only $372 \mathrm{mAh} / \mathrm{g}$ for graphite. Though silicon nanoparticles have remarkably high capacity, they suffer from rapid degradation with each cycle due to electrode volume expansion of approximately $400 \%$ during lithiation, placing a large strain on the material. With each cycle that strain creates cracks in the electrode particles and causes them to break down into smaller particles, which create void spaces between the particles and lead to poor contact as reflected in poor conductivity. In this review, we discuss exciting new research on silicon-based anodes conducted during the past couple of years. Besides stressing the importance of well-designed nanostructures of Si, we focus on optimization of the Si electrode and resulting performance enhancement by properly selecting binders and synergistically integrating them with various carbon materials during electrode design and fabrication. Importantly, although each improvement strategy has its own advantage, appropriate combination of them will yield much higher anode performance. We summarize the core issues in developing the silicon anode and effective strategies in yielding more promising results. 


\section{Content:}

1. Introduction

1.1. Lithium-ion battery

1.2. Graphite vs Silicon

2. Silicon Problems/Obstacles

3. Binder Effectiveness

4. Silicon Structure Effectiveness

4.1. Particle Size

4.2. Nanowires

4.3. Nanotubes

5. Silicon/Carbon Effectiveness

5.1. Carbon structures

5.2. Carbon Implementation

6. Conclusion 


\section{Introduction}

\subsection{Lithium-ion Battery}

Long-life, environmentally friendly, and low-cost reliable batteries are greatly needed to meet crucial energy storage demands of modern society. The lithium-ion battery, one of the most predominant power sources for mobile phones, laptop computers, and many other portable electronic devices, is increasingly being investigated for use in electric vehicles [1-5]. Since the commercialization of the lithium-ion battery by SONY in 1991, there has been a growth in its use, with expectations of continued growth $[1,6,7]$. Lithium is the third lightest element and has the lowest reduction potential of all known elements, $-3.04 \mathrm{~V}$ relative to the standard hydrogen potential. Unsurprisingly, lithium-ion batteries offer the most near-term promise for developing high energy and high power batteries to satisfy the future needs of society [7]. Among the many explored electrochemical power sources, these batteries are considered to have the greatest promise for use in large-scale applications. Research has thus been focused on improving their efficiency, robustness, and specific charging/discharging capability. Such research has included thermal characterization due to the potential for thermal runaway [8], virtual prototyping by dynamic modeling [9], and development of methods for recycling of used battery materials [10]. Figure 1 presents a basic schematic of the lithium-ion battery [7], using graphite as the anode and cobalt oxide as the cathode. 


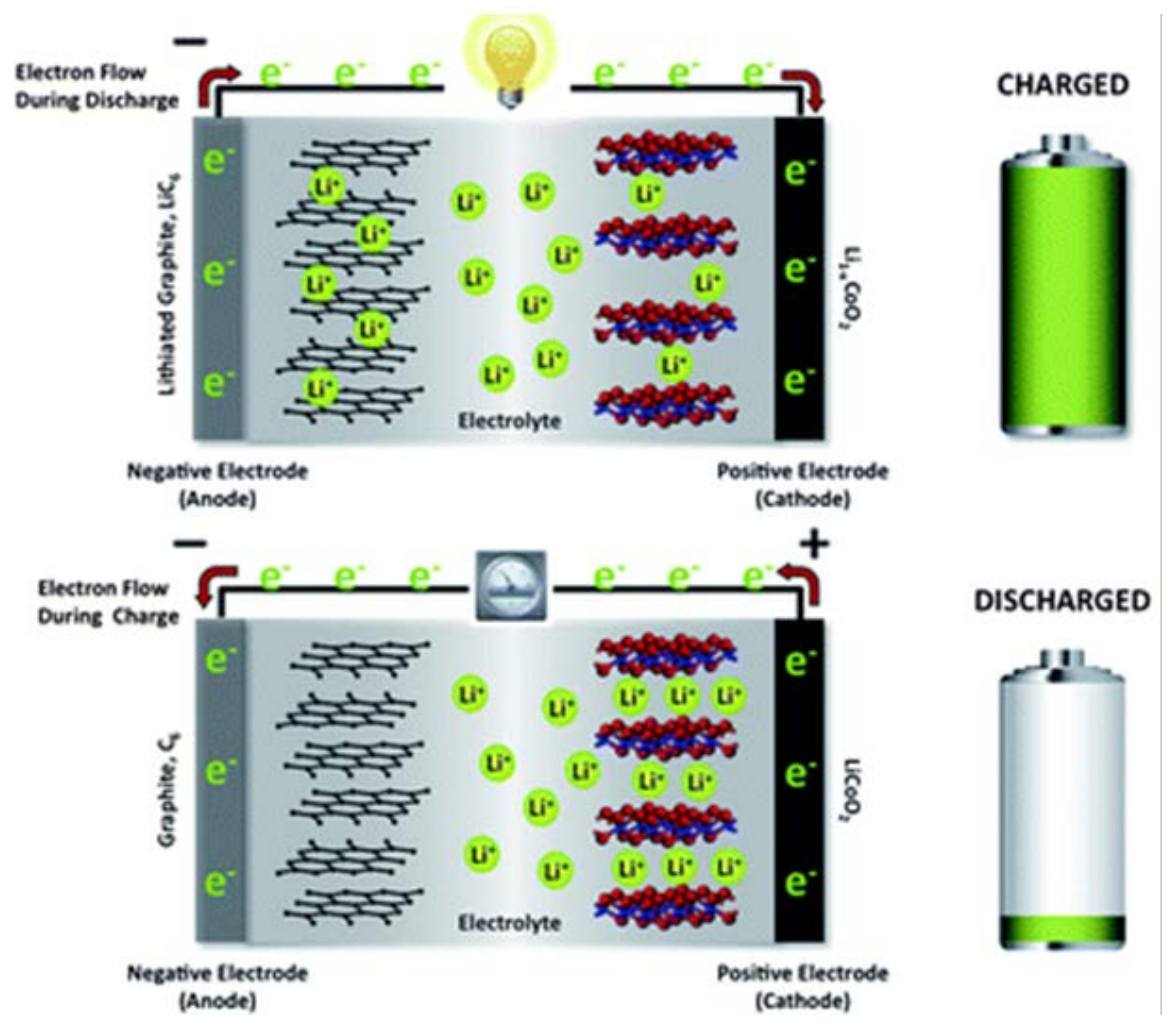

Figure 1: A schematic illustration of the working principles of a $\mathrm{Li}_{x} \mathrm{C}_{6} / \mathrm{Li}_{1-\mathrm{x}} \mathrm{CoO}_{2}$ cell. During discharge, lithium ions diffuse from a lithiated graphite structure (the anode) into a de-lithiated metal oxide structure (the cathode) with concomitant oxidation and reduction of the two electrodes, respectively. The reverse process occurs during charge. Reprinted with permission from Ref. [7]. Copyright 2012, Royal Society of Chemistry.

Generally, a lithium-ion battery consists of an anode and a cathode on two separate current collectors. Also, the electrolyte allows lithium ions to travel between the electrodes, and a separator keeps the anode and the cathode from making direct contact [1-3, 9-11]. The cathode usually consists of a metal oxide, and the anode tends to be a carbon material. Lithium-ion batteries charge and discharge through a process of lithiation (lithium insertion) and de-lithiation (lithium extraction) by means of electrochemical reactions. In this process, lithium ions diffuse 
back and forth through the electrolyte between the anode and the cathode. During de-lithiation (discharging) the lithium bonded with the anode material breaks apart, producing lithium ions and electrons, where the lithium ions travel and bond with the metal oxides on the cathode side and the electrons produce the electrical energy that power devices. During lithiation (charging), the lithium metal oxides from the cathode break apart, producing lithium ions that pass through the electrolyte and bond with the material on the anode side with the addition of electrons.

\subsection{Graphite vs. Silicon Anodes}

Graphite, currently the primary anode material used in commercial lithium-ion batteries, has a theoretical gravimetric capacity of $372 \mathrm{mAh} / \mathrm{g}[3,7,12-15]$. This active material is highly used because of its low and flat voltage plateau at about $0.1-0.2 \mathrm{~V}$ during discharge and its low and reasonable price [13-15]. Graphite also displays great stability and high capacity retention during cycling. However, besides the low capacity of graphite, some complications hinder its potential for use in advanced batteries. For example, graphite is sensitive to certain electrolytes. Ethylene carbonate and propylene carbonate are some commonly used solvents for the electrolyte in lithium-ion batteries. While ethylene carbonate-based electrolytes are always used with graphite anodes in lithium-ion batteries, propylene carbonate-based electrolytes are not compatible with graphite anodes because the propylene carbonate decomposes on the graphite surface, accompanied by graphite exfoliation [14, 16-18].

Anodes tend to consist of three parts: the active material, the conducting material, and the binder. Traditionally, the active material is usually graphite (with a stoichiometry of $\mathrm{LiC}_{6}$ ), the conducting material is a carbon material like acetylene black, and the conventional binder is polyvinylidene fluoride (PVDF), all uniformly dispersed in an N-methyl-pyrrolidone (NMP) solvent. The NMP solvent is needed because PVDF binder dissolves in only NMP and not water. 
Since the capacity of graphite is low for large-scale applications like electric vehicles, there has been a push to develop new and better anode materials with a high capacity, preferably at low potential. As a benchmark, the next-generation anodes need to have at least a stable specific capacity of $1000 \mathrm{mAh} / \mathrm{g}$ [19]. At present, silicon ( $\mathrm{Si}$ ) is potentially the best replacement for the graphite anode material because it has a high theoretical gravimetric capacity of $4200 \mathrm{mAh} / \mathrm{g}$ at very low potentials. The large difference in capacity between silicon and graphite arises because a silicon atom can bond with up to about four lithium ions (stoichiometry of $\mathrm{Li}_{4.4} \mathrm{Si}_{2} / \mathrm{Li}_{22} \mathrm{Si}_{5}$ ) while it takes six carbon atoms to bond with only one lithium ion [7, 13, 20-22]. Eq. 1 and Eq. 2 are the half reactions for the silicon and graphite anodes, respectively.

$$
\begin{gathered}
L i_{x} S i \leftrightarrow x L i^{+}+S i+x e^{-} \\
L i C_{6} \leftrightarrow L i^{+}+6 C+e^{-}
\end{gathered}
$$

Despite its promise, silicon is not being utilized in lithium-ion batteries because of mechanical issues that have become a roadblock. In this review, we address these issues and discuss recent research that has been conducted to overcome them. We also discuss where we stand on current innovations, and how we can apply them.

\section{Problems with Silicon Anodes}

As good as silicon's performance potential is for advanced lithium-ion batteries, there are some complications involving silicon's behavior. The problem lies with silicon's tendency to expand approximately $400 \%$ of its original size during lithiation, then reducing to a varying size during de-lithiation. The $400 \%$ expansion is a figure found using imaging technology to confirm and record different Li-Si phases with increasing lithium content [23-25]. It should be noted that silicon isn't the only material that suffers from such volume expansion; other high capacity 
material such as tin $(\mathrm{Sn})$, germanium $(\mathrm{Ge})$, and antimony ( $\mathrm{Sb}$ ) have the same volume change issue [26-30]. This expansion produces stress and strain on the silicon particles, causing cracks and breakage. The process of the silicon breaking down is known as pulverization. Due to this pulverization, electrical isolation of silicon fragments causes a loss in contact with neighboring fragments. In addition, the space created from the expansion pushing surrounding conducting material away from the active material also causes a loss of contact, resulting in low electrical conductivity. Without strong electrical contact with the current collector, the silicon fragments are not lithiated or able to contribute to the battery's capacity. This behavior yields low capacity stability and rapid capacity degradation over a number of cycles. Figures $2 \mathrm{a}$ and b [31] illustrate how pulverization affects the silicon particle and how the expansion reduces the conductivity from the loss of contact with neighboring particles.

(a)

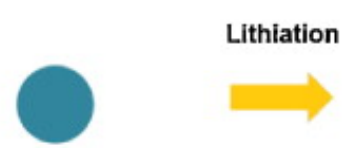

(b)
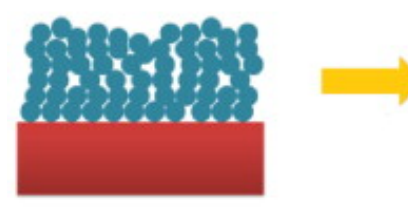
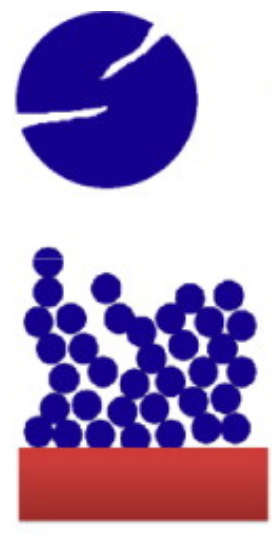

(c)

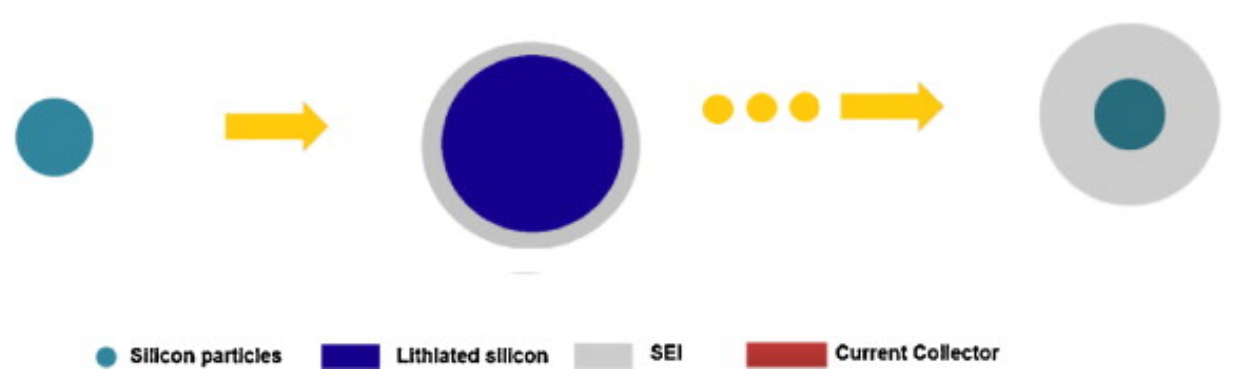


Figure 2: Mechanisms of Si electrode failure: (a) material pulverization, (b) morphology and volume change of the entire Si electrode, and (c) continuous SEI growth. Reprinted with permission from Ref. [31]. Copyright 2012, Elsevier Ltd.

Another issue that contributes to the fast anode degradation is the solid-electrolyte interface (SEI). The SEI forms on the surface of the electrode during the first lithium insertion phase and works like a barrier between the electrolyte and the electrode. It is formed from the solvent and the decomposition of the salt in the electrolyte. This barrier allows lithium-ion conduction and is an insulator to electron flow, which limits further electrolyte decomposition and prevents the cycle performance of the lithium-ion battery from dropping continuously. Battery performance, irreversible charge "loss", rate capability, and cyclability are highly dependent on the quality of the SEI $[32,33]$.

Some commonly used electrolytic salts are $\mathrm{LiPF}_{6}, \mathrm{LiAsF}_{6}, \mathrm{LiBOB}, \mathrm{LiClO}_{4}$, and $\mathrm{LiBF}_{4}$, with $\mathrm{LiPF}_{6}$ being the most often reported in the literature. These electrolytic salts are usually dissolved in a mixture of carbonate solvents such as ethylene carbonate, dimethyl carbonate, diethyl carbonate, or propylene carbonate, but as mentioned earlier, propylene carbonate is less effective as an electrolyte solvent compared to the others, so it is the least reported [32, 34]. The irreversible charge "loss" with these electrolytes has to do with the lithium ions lost during the formation of the SEI. This loss causes the capacity drop after the first cycle and is the reason why the initial coulombic efficiency starts out so low. The amount of SEI formed is also relative to the surface area of the anode material in the electrode. While the silicon anode gets more pulverized after each cycle, the surface area continues to increase. Since the SEI forms on the surface of the electrode, greater surface area leads to more SEI formation during the lithium 
insertion in each cycle. Since silicon constantly breaks down from pulverization with almost each cycle, the greater surface area consumes more lithium ions from the electrolyte to form a thicker SEI layer, accompanying by constant capacity drop. Figure $\mathbf{2 c}$ shows how the SEI grows thicker over long sets of cycles because of the fresh surface that becomes constantly exposed.

In general, the stability of the SEI layer is dependent on the composition of the electrolyte, salt, solvent, and electrolyte additives since it is the decomposition of these components that forms the SEI. As mentioned earlier with graphite, propylene carbonate as a solvent for the electrolyte can cause the graphite to exfoliate. This effect could cause the SEI to become unstable and lead to poor performance, and this problem could potentially be the same with silicon to a degree. The cause of the unstable SEI for the silicon anode could mainly be caused by the damage to the SEI layer from expansion. However, it should also be acknowledged that there are potential issues with the surface chemistry of the silicon particle. Electrolytes and additives are being developed to help make the SEI more stable and possibly more suitable for silicon surface interaction [35, 36]. For example, Nie et al. and Etacheri et al. studied the effects of an additive/co-solvent, called fluoroethylene carbonate (FEC), to the traditional ethylene carbonate (EC) solvent [37, 38]. A high concentration of FEC in the electrolyte generates an SEI composition with a high concentration of $\mathrm{LiF}$ and a polymer that may be a polyalkene or polycarbonate, while a low concentration of FEC has little effect on the SEI composition. Unfortunately, even with the addition of FEC, the SEI on the silicon anode is still not as stable as the SEI on graphite anode. Nevertheless, the FEC is able to mitigate the capacity fading of $\mathrm{Si}$ anodes, as shown in Figures 3a and 3b [38]. 

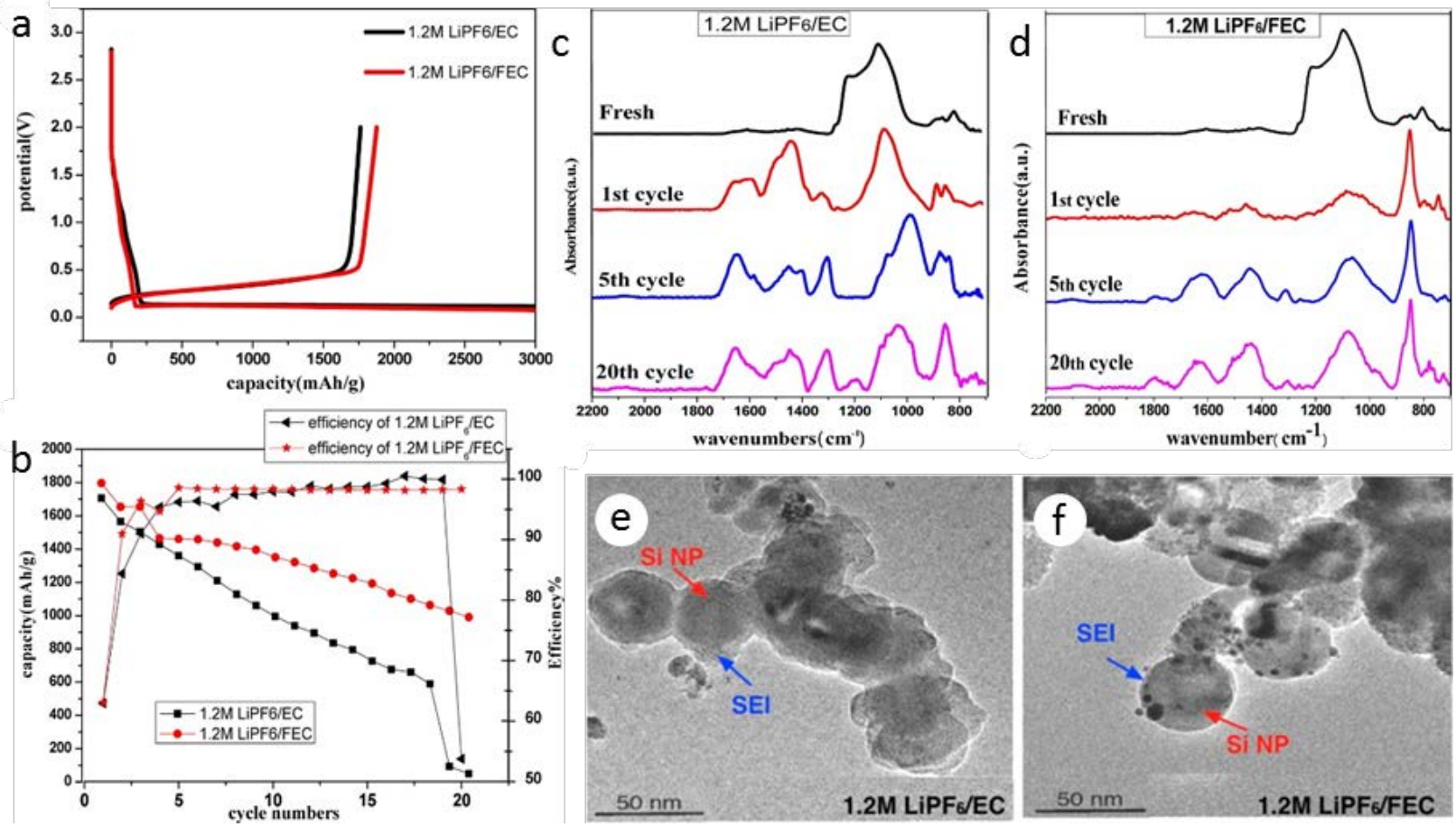

Figure 3: (a) First-cycle charge/discharge profiles of half cells with BF (binder free)-Si electrodes and electrolytes containing EC and FEC. (b) Cycling performance of EC- and FEC-containing electrolytes in BF-Si cells at 20 cycles. Infrared spectra of BF-Si electrodes cycled with (c) EC electrolyte and (d) FEC electrolyte (from top: fresh electrode, 1st, 5th, and 20th cycles). TEM images of silicon nanoparticles in (e) EC electrolyte and (f) FEC electrolyte. Reprinted with permission from Ref. [38]. Copyright 2013, American Chemical Society.

As troubling as the problem is, the damage caused by the pulverization of the silicon particles can be reduced by several methods, such as use of more effective binders, improvement of the silicon particle structure, and use of novelty carbon structures. The effect of each of these methods on performance is reviewed below. Though each of these can help stabilize the performance of silicon anodes, determining their significance could help us discover more effective ways to utilize them. 


\section{Binder Effectiveness}

Binders hold the electrode together, just as the name suggests. The use of different binders in order to improve electrode performance has been investigated for over a decade. Some binders have been developed and synthesized, such as mussel-inspired adhesive [39], in an attempt to replace traditional binders. The traditional binder used in many graphite anode batteries is PVDF. Unfortunately, this binder cannot accommodate the volume expansion of the silicon material because of its weak van der Waals forces [40]. A number of experiments have shown that silicon electrodes made with PVDF binder can barely maintain capacities above 1000 $\mathrm{mAh} / \mathrm{g}$ for only a few cycles [40-44]. PVDF has been the binder of choice in most research in the last three decades. Since mid-late 2000s, methods to alter or modify PVDF have been used to improve the performance of lithium-ion batteries, including those with silicon anodes [40]. For example, Li et al. tried heat treatment of PVDF-based Si anodes in argon at $150-350{ }^{\circ} \mathrm{C}$ and found that this method helped improve performance, yielding a specific capacity of approximately $600 \mathrm{mAh} / \mathrm{g}$ after 50 cycles. Compared to the case without the heat treatment, this capacity is a significant improvement [43]. Also, Chen et al. used cross-linking of PVDF-based $\mathrm{Si}-\mathrm{Sn}$ anodes and found that this method increased the binder elastic modulus and strength while simultaneously improving the anode's stability [42]. Though the efforts were fruitful, the stabilized capacities were still insufficient for practical applications. Even with improvements to the PVDF binder, it still has shortcomings and is incapable of effectively dealing with silicon's volume expansion upon cycling.

New and effective binders are being researched and in use in silicon-based anodes to better compensate for the volume expansion. Two binders with good potential that have shown greater stability in silicon-based anodes are poly(acrylic acid) (PAA) and carboxymethyl 
cellulose (CMC). What makes these binders good for silicon-based anodes is that they have a high elastic modulus in comparison to that of PVDF. It is reported that the Young's moduli of PAA and PVDF, respectively, are 4000 and $650 \mathrm{MPa}$. In addition, Na-CMC has a Young's modulus close to that of PAA [40]. The higher elastic modulus results in lower odds of deformation, which makes them more effective in containing the volume expansion for more cycles and maintains better contact with all the anode materials, even if silicon continues to break down. However since PAA and CMC are quite brittle, they aren't functional as elastomeric binders [40, 41]. Regardless what makes them truly attractive binders is their chemical interaction with the silicon surface since their carboxyl functional groups interact strongly with $\mathrm{SiO}_{2}$, which is present on the surface of the silicon particles via hydrogen bonding $[40,45,46]$. There is also an appeal to the use of PAA and CMC as a binder in that they dissolve well in water while PVDF only dissolves in NMP. The use of water as a solvent compared to NMP is more favorable since water is more environmentally friendly, more abundant, and inexpensive. While water can cause surface oxidation of the silicon, PAA can also be dissolved in an organic solvent such as ethanol to avoid the issue. There has been some work on binder mixtures, one of which is CMC and styrene butadiene rubber (SBR) [47, 48]. In one study, an electrode having a binder content of $1 \%$ SBR and $1 \%$ CMC had the same cycle stability as an identical electrode containing 10\% PVDF binder [49]. Other experiments in checking the effects of electrolyte solvent on binders suggest that PVDF binder in electrolyte solution behaves as an easily deformable material, with small resistance to both elastic and plastic deformations. On the other hand, PAA and Na-CMC show little to no significant change in initial capacity [40]. Figure 4 [40] illustrates the difference in effectiveness among the three binders: PAA, CMC, and PVDF. 
Both PAA and CMC have similar performance, as expected since they have similar elastic properties, and PVDF falls short as evident from the performance loss within the first 2 cycles.
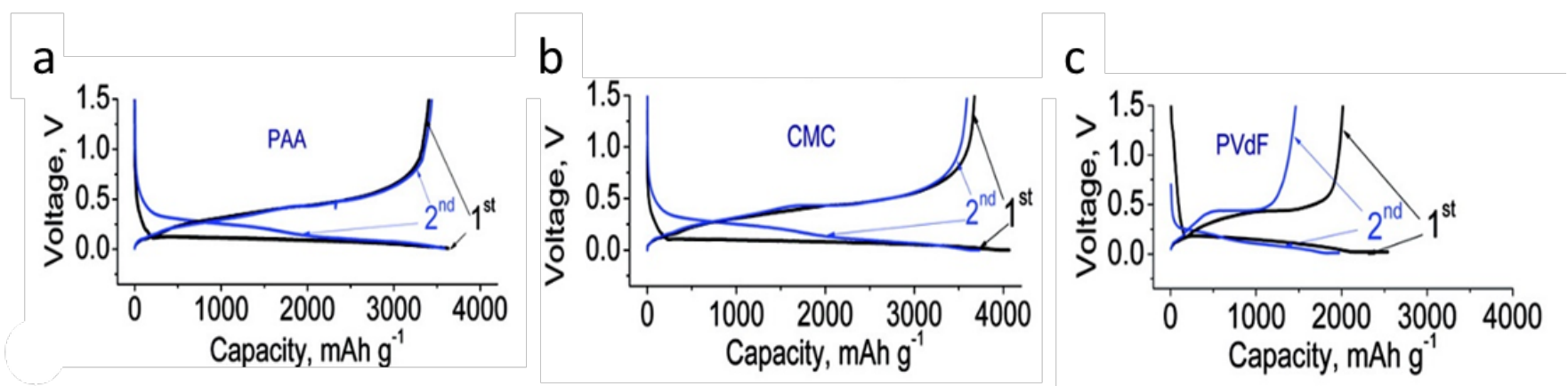

Figure 4: Galvanostatic charge-discharge profiles of Si-based anodes with different binders recorded at the $\mathrm{C} / 20$ rate: (a) PAA, (b) CMC, and (c) PVDF. Profiles reported for Si part of the anode only. Reprinted with permission from Ref. [40]. Copyright 2010, American Chemical Society.

Optimization studies have shown that higher binder content is related to lower fading capacity, but the overall capacity will be lower due to a lesser amount of actual anode material. With a lower binder content, initial capacity starts high but fades more rapidly [40]. Though the addition of binders with high elastic modulus is a key factor in the improvement of silicon-based lithium-ion batteries, it alone will not result in high capacity over long-term cycle stability. Further validation of binder effectiveness is still required for future $\mathrm{Si}$ anodes.

Currently, PAA and CMC are the binders of choice in many research efforts involving silicon anodes, but some studies are looking into different binders to improve silicon performance. Recently, in addition to PVDP and PAA, Erk et al. studied the binders alginic acid sodium salt (AA), polyethylene oxide (PEO), and poly(vinyl alcohol) (PVA). The group also investigated combination of binders and solvents using ethylene carbonate (EC), ethyl methyl 
carbonate (EMC), and fluoroethylene carbonate (FEC). Their purpose was to observe the effectiveness of each binder in conjunction with different electrolytes.
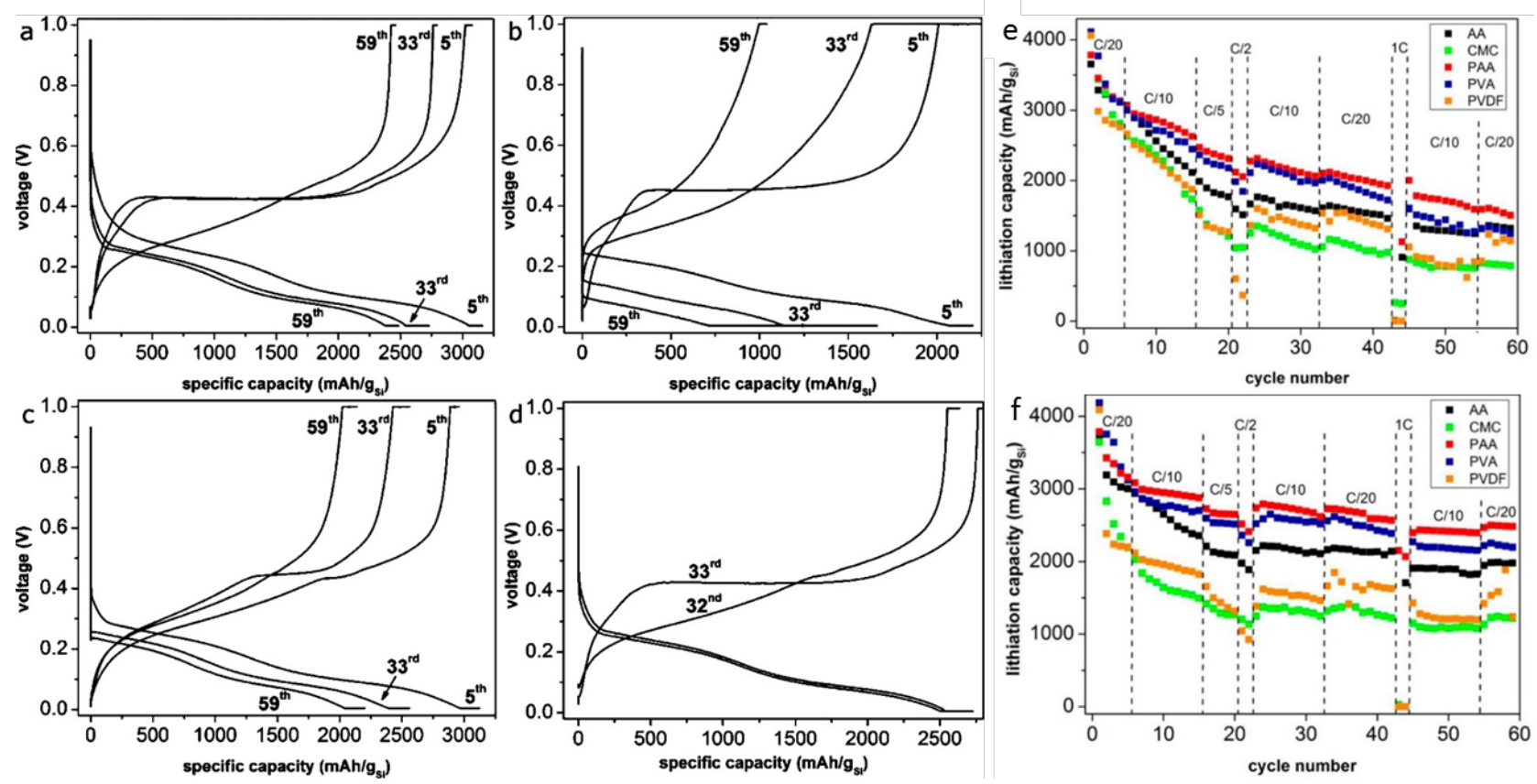

Figure 5: $(\mathrm{a}-\mathrm{c})$ Voltage profiles of electrodes prepared from (a) PAA, (b) PVDF, and (c) PVA at a rate of C/20. The electrolyte system used in these experiments was FEC/EMC. (d) Voltage profiles of the PAAbased electrode for the 32nd (C/10 rate) and 33rd (C/20 rate) cycles. (e-f) Lithiation (discharge) capacity as a function of cycle number and rate for electrodes comprising different polymer binders: (e) EC-based electrolyte and (f) FEC-based electrolyte. Reprinted with permission from Ref. [19]. Copyright 2013, American Chemical Society.

Figure 5 [19] shows the performance of each binder using EC and FEC electrolyte. Note that PEO is not included in the figure because it had caused significant capacity fading. Among the binders tested, PAA still seems to have the best performance, followed by PVA, AA, PVDF, and CMC. A noteworthy discovery was that FEC resulted in better performance retention than EC. 
This finding shows the significant role that the electrolyte solvent plays in performance. Most of the binders used, except for $\mathrm{CMC}$, resulted in similar performance increases in tests using FEC solvent. In the case of PAA, the specific capacity is approximately $1000 \mathrm{mAh} / \mathrm{g}$ higher for an electrolyte with FEC in comparison to EC. Figure 5 also [19] displays the charge-discharge voltage profile of the silicon anode using three of the binders: PAA, PVA, and PVDF. In these graphs we can see the voltage profile degradation with cycling. As expected, the degradation in the PVDF binder profile between cycles is greater than that of PAA and PVA.

As the binder is meant to help reduce the level of pulverization of the silicon particle and keep contact with surrounding conducting materials, there is also the possibility of removing the need for a conducting material. Research done by Liu et al. attempted to do just that by using a conductive binder [50]. Conductive binders take on the dual functionality of both the binder and conducting material. This would eliminate the issue of electrical connectivity and reduce mass required for conducting material. There are some drawbacks to the use of conductive binders. As an example, polyaniline (PANI), a commonly used conductive polymer, will not stay p-doped below a potential of $1 \mathrm{~V}$, causing a loss of electronic conductivity when it is used in the $\mathrm{Si}$ anode. To counteract this problem, Liu and his coworkers developed polymer binders that could be cathodically doped for high electronic conductivity under the reducing environment of the anodes [50]. Figure 6 [50] compares their method with the traditional method and shows the promotional role of the conductive polymers they developed as binders, referred to as poly $(9,9-$ dioctylfluorene - co - fluorenone) (PFFO) and poly (9,9 - dioctylfluorene - co - fluorenone - co methylbenzoic acid) (PFFOMB). Both PFFO and PFFOMB are based on polyfluorene (PF)-type polymers. Two key function groups, carbonyl $\mathrm{C}=\mathrm{O}$ and methylbenzoic ester $-\mathrm{PhCOOCH}_{3}(\mathrm{MB})$, 
were introduced for tailoring the lowest-unoccupied-molecular-orbit electronic states and for improving the polymer adhesion, respectively.
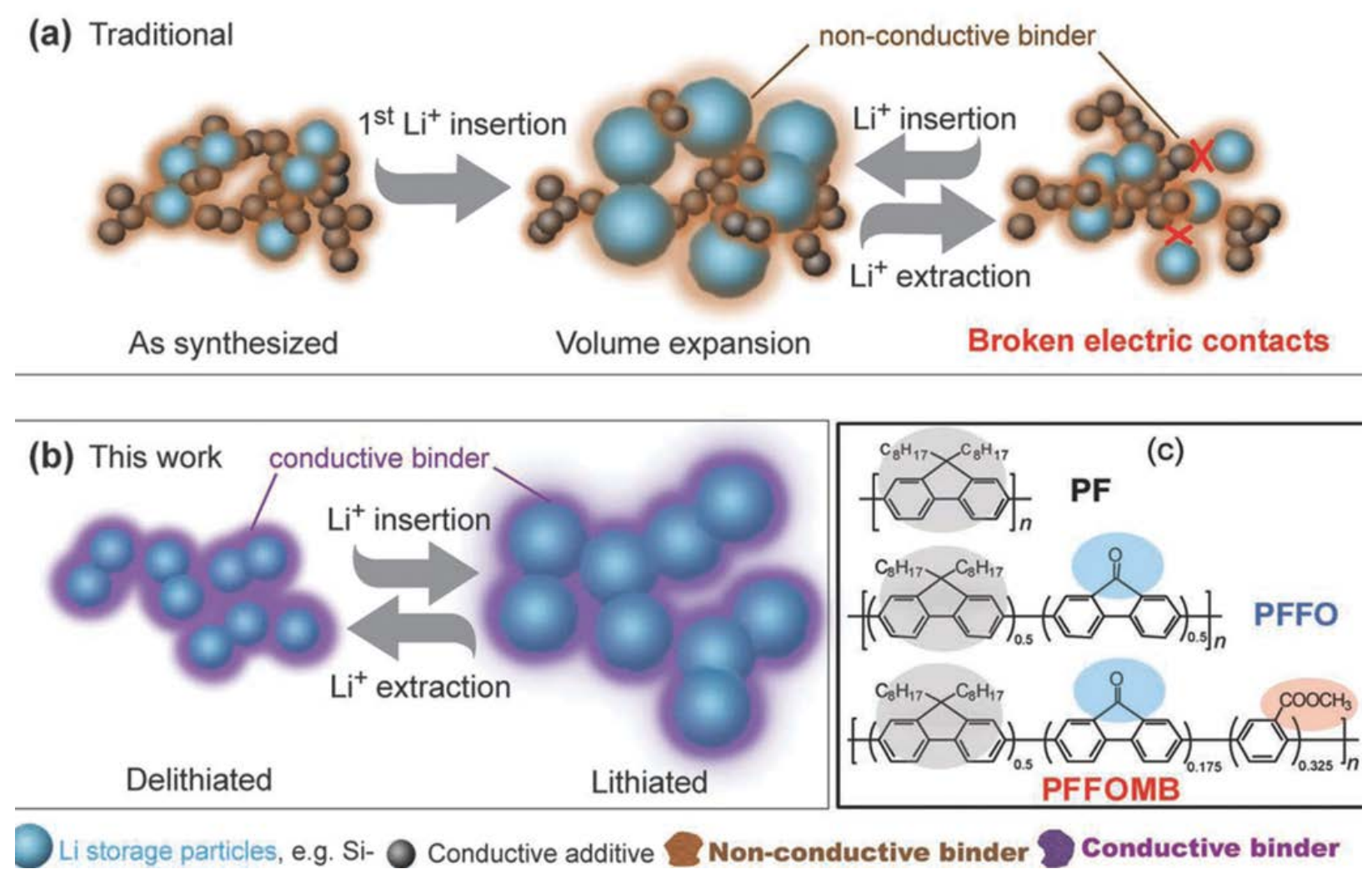

Figure 6: Schematics for improving the binder with functional groups to address the volume change in silicon nanoparticles. a) Failure of the traditional binder using a mixture of acetylene black (AB) and PVDF polymer. b) Maintenance of both electronic and mechanical integrity of the silicon nanoparticles electrode during cycling performance with dual-function conductive polymer. c) Structure of dualfunction conductive polymers. To improve the conductivity and mechanical binding force of the conductive polymer, two key function groups in poly(9,9-dioctylfluorene-co-fluorenone-comethylbenzoic acid) (PFFOMB), carbonyl and methylbenzoic ester were used to modify the polymer. Reprinted with permission from Ref. [50]. Copyright 2011, Wiley-VCH. 
Figure 7 [50] shows scanning electron microscopy (SEM) and transmission electron microscopy (TEM) images of the polymers at work before and after cycling, while also giving some data on the performance of the binders in comparison to other binders like PVDF and PANI, as well as the use of acetylene black (AB). Figure $7(\mathrm{~g})$ shows that all the silicon anodes with binder except that with the PFFOMB have high capacity fading within the first few cycles. Such results were expected with PVDF and PANI. Figure 7(a) presents an adhesion force plot, showing that PFFOMB has a much higher binding force compared to PFFO. The reason is that the methylbenzoic ester portion added to the PFFO improves the binder's chain flexibility. This flexibility allows the anode to withstand the silicon's expansion, resulting in continuous electrical conductivity.
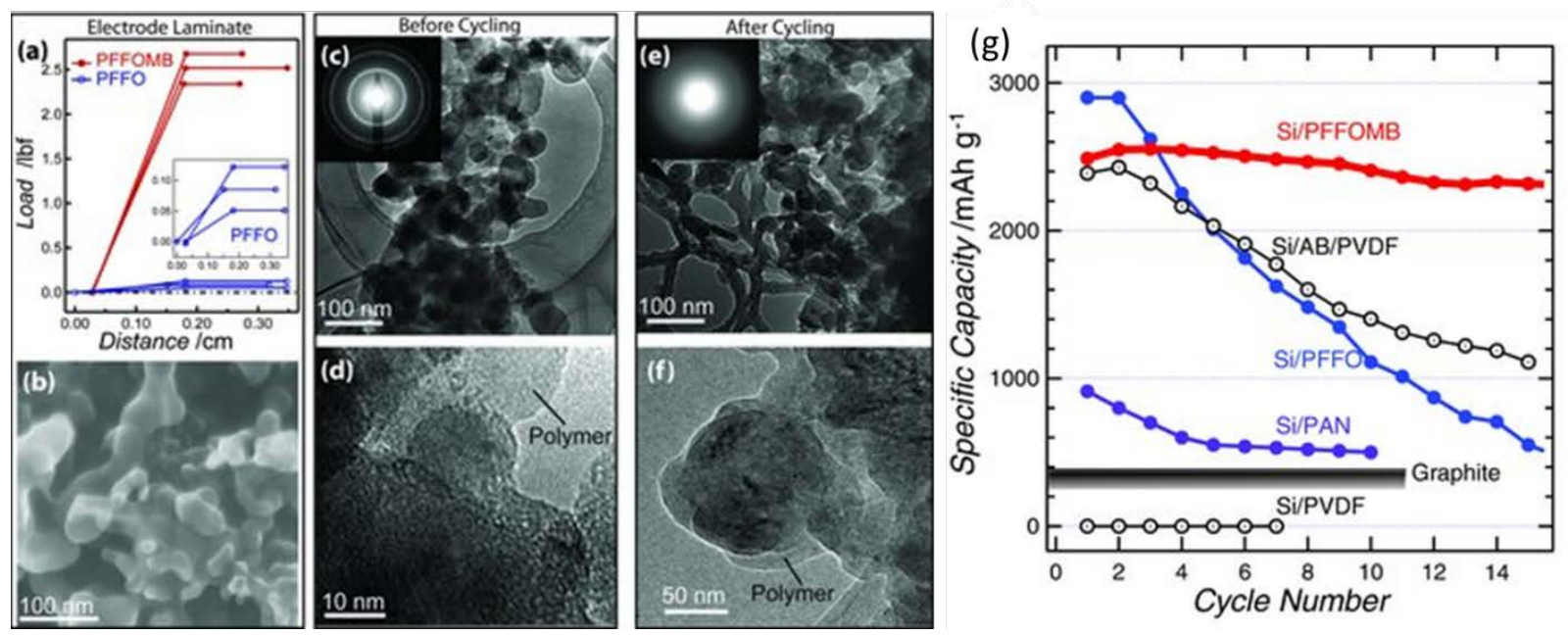

Figure 7: Physical properties of the Si/PFFOMB composite electrode. (a) Adhesion force plot of the Si/polymer electrode laminates. Insert shows the amplified PFFO data. (b) SEM image of a standard porous microstructure of the surface of the Si/PFFOMB composite electrode. (c-d) TEM images of Si nanoparticles embedded in the as-prepared PFFOMB polymer matrix. (Inset) Electron diffraction pattern shows the crystalline nature of Si before cycling. (e-f) TEM images of Si nanoparticles maintained in the PFFOMB polymer matrix after 32 cycles. (Inset) Electron diffraction pattern shows the loss of 
crystallinity of $\mathrm{Si}$ after cycling. (g) Initial cycling behaviors of $\mathrm{Si}$ particles in different conductive matrixes against lithium metal counter electrodes at C/10 rate. Reprinted with permission from Ref. [50]. Copyright 2011, Wiley-VCH..

The above studies indicate several requirements to developing an improved binder for the silicon anode. First the binder should have high elasticity. PVDF generally lacks this high elasticity and is prone to succumb to plastic and elastic deformation. Without altering the binder through chain linking procedures, it undergoes the loss of conductivity throughout the electrode. In contrast, PAA and $\mathrm{CMC}$ have considerably higher elastic moduli, so that electrodes using these binders tend to have a less rapid fading of capacity. Also, being able to withstand the force of the silicon's expansion reduces the effect of pulverization. On the other hand, PAA and CMC are relatively brittle binders, meaning that they have minimal elongation and cannot function well as elastomers. Another possible factor is the contact strength of the binder with the silicon. The carboxyl group on PAA and CMC has a strong interaction with the $\mathrm{SiO}_{2}$ on the particle's surface, improving the chance of maintaining contact between the silicon particles. It is also better if a binder is strong enough that less binder is necessary in terms of ratio in the anode as it would allow for higher capacity. In general, the binder primarily addresses the issue of electrical contact of the entire electrode, but does not address the pulverization issue as such. Considering that some binder-free electrodes have been developed [51-53], this means that binders are not exactly necessary but are convenient in that they are more easily prepared in many slurry-based electrode preparations. In other cases, alternative materials from even living organisms can show promises. Research from Kovalenko et al. used alginate, a natural polysaccharide extracted from brown algae, mixed with silicon nanopowder was able to achieve a stable battery anode 
possessing reversible capacity eight times higher than that of the state-of-the-art graphitic anodes

[54]. This is also partly contributed by the formation of strong hydrogen bonding between the hydroxylated Si surface and alginate carboxylic moieties similarly to bonding of PAA and CMC mentioned earlier.

\section{Silicon Nanostructure Effectiveness}

Commercial silicon usually comes in powder form with particle sizes ranging from micro- to nano-scale. Commercially available silicon powder is typically covered by a native oxide layer and further terminated with hydroxyl groups. During synthesis, such a shell is readily formed because of the presence of trace amounts of oxygen and water. As a result, most silicon microand nano-scale powders can be processed under ambient atmosphere because the surface oxide acts as a passivating layer [19]. Given that silicon particles have poor performance by themselves for reasons that we have already stated, it is possible for silicon performance to be improved by changes in structure. Some quick examples of silicon structures that have been studied include interconnected hollow nanospheres whose hollow structure allow for large strains without pulverization compared to solid particles [55]. Mesoporous silicon uses the mesopores of varying sizes as a buffer zone for the volume expansion. This can also work to limit the volume expansion to prevent pulverization [56-58]. Silicon thin films, though a common means to apply silicon material, is not easy to form thick film, which limits the practical application of silicon without some additions but is more inexpensive to process $[59,60]$. A core-shell structure that uses a rigid skeleton support silicon in a $\mathrm{Si} / \mathrm{B}_{4} \mathrm{C}$ composite which alleviated the volume expansion [61]. Silicon nanowires [62-66] and silicon nanotubes [22, 67] use cylindrical shapes to reduce stress by apply expansion strain more radially and allow better strain relaxation. Several very insightful review articles, published in the last few years, discuss the role of 
nanostructure control in improving Si anode performance [31, 68-71]. Here, we only discuss a few of these structures in more detail to highlight their benefits and address aspects of the problems that they cause with silicon particles. Whether a change in structure is enough to nullify the effects of the problem will be addressed here.

\subsection{Particle Size}

The particle size of the silicon particle can play a role in how quickly the battery performance declines. Smaller particle sizes are much more electrochemically stable compared to larger particle sizes. With larger size particles, high initial specific capacities are achievable but tend to fade rapidly. The smaller size of nano-sized particles makes them more stable because they reduce mechanical stress from the volume expansion [23, 72]. In fact, research shows that pulverization doesn't occur to nano-particles in certain size range. At a critical particle size of approximately $150 \mathrm{~nm}$, particles exhibit no cracks or fractures below this size [73-75]. Figure 8 [23] shows the galvanostatic charge-discharge profile of both micro- and nano-sized silicon particles, proving the effect of the change in particle size. For the micro size, the capacity drops more than $2000 \mathrm{mAh} / \mathrm{g}$ after the first cycle. For the nano size, the drop after the first cycle is much less and capacity fading is less rapid, still maintaining at least $1000 \mathrm{mAh} / \mathrm{g}$ after 22 cycles. These results aren't always the case for silicon nanoparticles depending on how the electrode is prepared. In the anode tests in Figure 8, the weight ratio of nano-Si, carbon black, and PVDF binder was 4:4:2. Using less silicon and more carbon black increases the electrical conductivity of the electrode in comparison to a typical weight ratio of 6:2:2 widely used by most of researchers. In the case of the micro particles, the weight ratio is actually $8: 1: 1$. This difference is most likely a big contributor to the large performance drop, but the micro particle size will always yield a more rapid fading of capacity to below $1000 \mathrm{mAh} / \mathrm{g}$ within at least 10 cycles [23, 
72]. Since the nanoparticle size is smaller, so too is the void space created by the volume expansion. This condition minimizes the destruction of the conducting network around the silicon and the electrode. There is also a factor of loading that can affect overall performance as electrodes with lower loading, especially very low loading, tend to show better specific capacity.
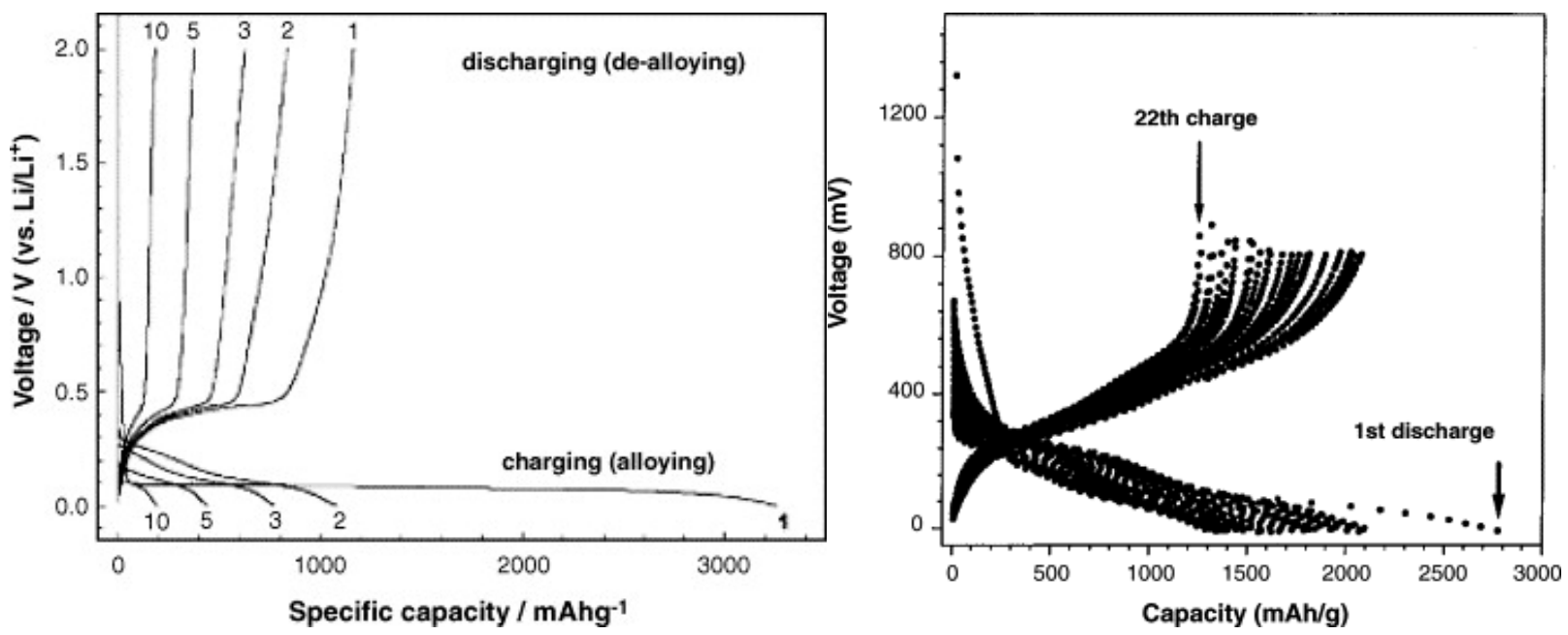

Figure 8: Galvanostatic charge-discharge profile of the silicon anodes with micro (left) and nano (right) size particles. Reprinted with permission from Ref. [23]. Copyright 2007, Elsevier Ltd.

The difference in particle size and performance can also still apply within the nano size range. In this range, the smaller particle size still performs better than the larger size. Figure 9 [19] shows the performance of electrodes made with 50-nm and 100-nm silicon particles. In Figures 9(a-f), TEM, energy-filtered (EF) TEM, and high-resolution (HR) TEM images of these particles are also displayed to illustrate the difference in their nanostructures. Regardless of their different particle sizes, their capacities are both higher than $1000 \mathrm{mAh} / \mathrm{g}$ for cycle numbers less than 30 . This finding further reinforces the idea of using silicon nano- over micro-particles, since they are more electrochemically stable. 

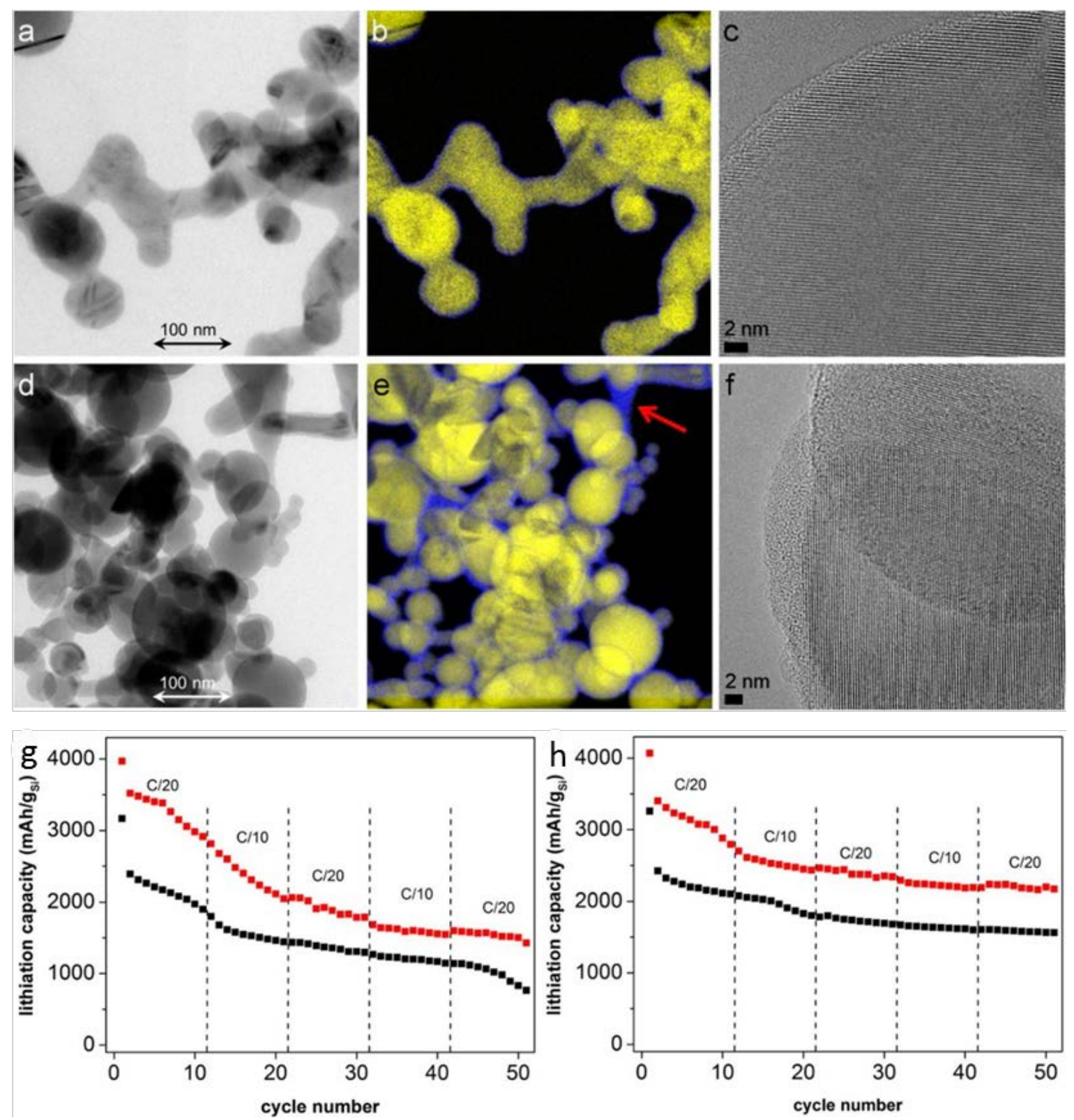

Figure 9: Microstructure of (a-c) Si-50 nm and (d-f) Si-100 nm powders. (a, d) Bright-field TEM images. (b, e) EFTEM mapping of the areas shown in panels a and $d$. The red arrow in panel e indicates an oxygen-rich domain. (c, f) HRTEM images. (g,h) Lithiation (discharge) capacity as a function of cycle number for electrodes prepared from Si-50 nm (solid squares in red) and Si-100 nm (solid squares in black) powders and AA as binder with (g) EC-based electrolyte and (h) FEC-based electrolyte. Reprinted with permission from Ref. [19]. Copyright 2013, American Chemical Society. 


\subsection{Silicon Nanowires}

A proposed anode structure is silicon nanowires. Produced by Chan et al., silicon nanowires were grown by a vapor-liquid-solid (VLS) process directly on stainless steel current collectors to ensure strong contact $[66,76]$. The three main benefits of the use of silicon nanowires are the accommodation of expansion, strong electrical contact, and elimination of binder. Nanowire-based electrodes can accommodate volume expansion during cycling while withstanding the strain that would cause pulverization. This is because the facile strain relaxation in the nanowires allows for expansion in the wire diameter without cracks or breaks forming. In addition, the nanowires are grown on current collectors, which create a strong electrical contact $[21,66]$ that allows for continuous and efficient electron transport throughout each wire. This condition ensures that all connecting nanowires contribute to the capacity because the expansion does not cause neighboring silicon nanowires to lose contact as the micro-particles seen in Figure 2b. Figure $10[31,66]$ further summarizes the work of Chan et al. on silicon nanowires. Figures 10 (a-b) illustrate the pulverization problem of using film and particle application of silicon electrodes. As shown in Figure 10b, when using the nanowires there is no breaking down of the anode material, for reasons mentioned earlier. 

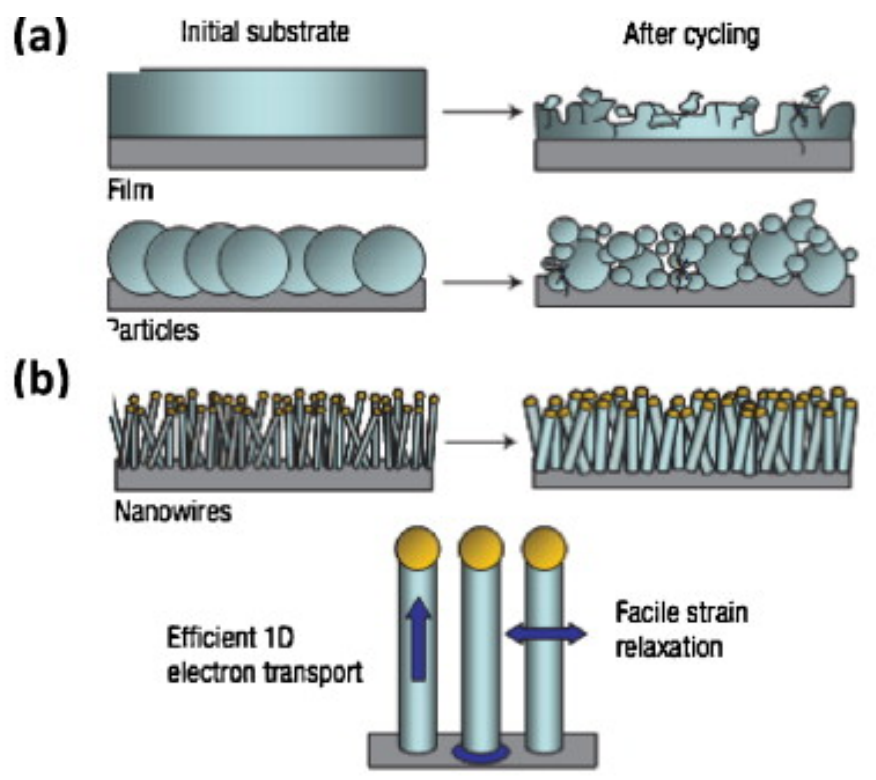

Good contact with current collector
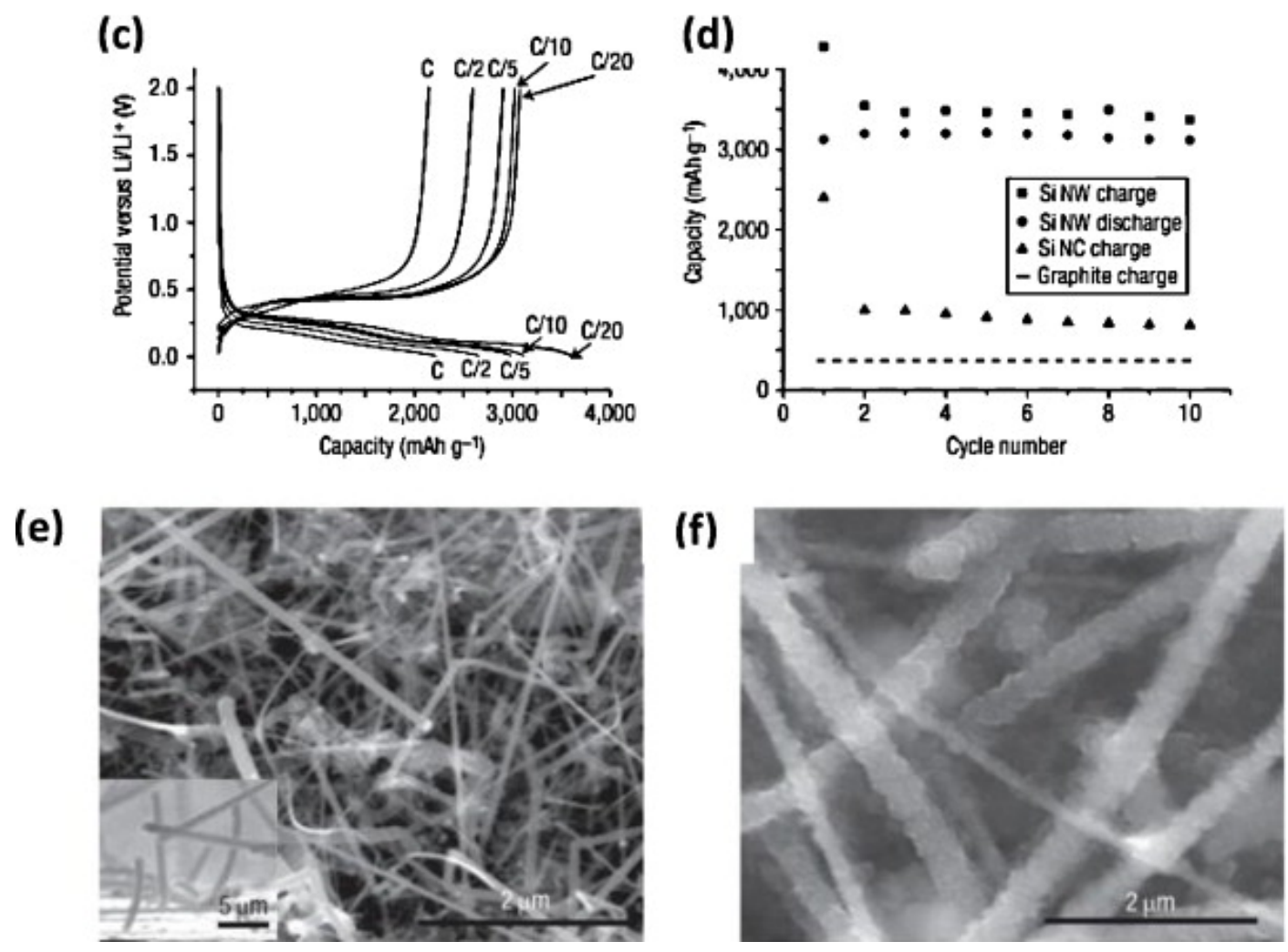

Figure 10: (a) Schematic of morphological changes that occur in Si during electrochemical cycling. The volume of Si anodes changes by about $400 \%$ during cycling. As a result, Si films and particles tend to pulverize during cycling. (b) Schematic of morphological changes that occur in Si nanowires grown 
directly on the current collector, which do not pulverize or break into smaller particles after cycling. Rather, facile strain relaxation in the nanowires allows them to increase in diameter without breaking. (c) Voltage profiles for the Si nanowires cycled at different currents. (d) Capacity versus cycle number for the Si nanowires at the C/20 rate. (e and f) SEM image of pristine Si nanowires before (e) and after (f) electrochemical cycling. Reprinted with permission from Ref. [31, 66]. Copyright 2012, Elsevier Ltd.

Figures 10 (c-d) show that silicon nanowires have high discharge capacities and stable cycling over tens of cycles, with reversible capacities as high as approximately $3400 \mathrm{mAh} / \mathrm{g}$ [21]. It is likely that the ability to maintain form without pulverization during cycling is what contributes most to this high level of stable capacity. Since pulverization is the main drawback to silicon particles due to the constant development of SEI layers on newly formed surfaces, which causes the constant drop in capacity, nanowires hold high promise to address the pulverization issue. The growth of the nanowires on the current collector for good contact also eliminates the need for binders, which would add unnecessary weight [66]. However, there is a disadvantage to this method when planning for large-scale application: fabricating the silicon nanowires using the VLS method in large quantities is very complex, leading to a high manufacturing cost [50, 63]. Research was done on a more efficient synthesis method using a supercritical fluid-liquid-solid (SFLS) slurry, but results did not match those of the VLS method [63].

Another potential hindrance is the issue of the SEI layer. Though the nanowire structure helps reduce cracks in the anode material during charging, it doesn't prevent the expansion. The SEI layer can still be broken after the expansion and leave some exposed surfaces for new SEI layers to form. After repeated cycles, a very thick SEI layer will likely be formed, causing a loss of lithium ions and limited ion transfer through the SEI layer [65].

\subsection{Silicon Nanotubes}


With a similar structure to the nanowire, silicon nanotubes have also been investigated. Like nanowires, they expand radially from the center except instead of being a solid cylinder shape, they are in the shape of a tube. The purpose behind the tube shape is to increase the surface area to which the electrolyte is exposed. Park et al. fabricated a silicon nanotube structure to overcome the problem that the limited surface area of some silicon structures causes the anode material to be more polarized at high current rates, leading to capacity fading [22]. The result of their research yielded a nanotube electrode showing a reversible capacity of about $3200 \mathrm{mAh} / \mathrm{g}$ with high capacity retention of approximately $89 \%$ for 200 cycles [22]. There is still the issue of the SEI layer on the nanowire anodes, since this structure doesn't prevent silicon expansion, but Park et al. compensated for this shortcoming by adding a carbon coating on the tube to stabilize the SEI film. Wu et al. tested a double-wall method with the silicon nanotube that used silicon oxides $\left(\mathrm{SiO}_{\mathrm{x}}\right)$ as a confining layer around the outer wall of the nanotube. Their double-walled silicon nanotube (DWSiNT) allows for lithium ions to pass through the $\mathrm{SiO}_{\mathrm{x}}$ layer while preventing electrolyte from coming into contact with any cell component besides the $\mathrm{SiO}_{\mathrm{x}}$ layer. Since the confining layer of $\mathrm{SiO}_{\mathrm{x}}$ is mechanically rigid, during lithiation the silicon nanotube will expand inwards only. With this expansion, the SEI layer will remain intact, preventing fresh surfaces from being exposed and a new layer of SEI from forming. Figures 11(a-c) [67] demonstrate the benefits of the $\mathrm{SiO}_{\mathrm{x}}$ layer and show how it allows for a more stable SEI layer. Figures 11(d-f) show SEM and TEM images of the double-walled silicon nanotubes. Figures 11(a, b) show the damage to the SEI layer caused by the silicon expanding outwards in the case of a silicon nanowire and a silicon nanotube. This expansion also applies to other silicon nanostructures that don't have some kind of mechanism to prevent volume expansion. The SEI film will continue to form until a thick enough layer is formed to be unaffected by the expansion. 
In Figure 11(c), a clamping layer is surrounded by the SEI film, and during charging the expansion occurs inward without doing any harm to the film. This layer can also be substituted with a carbon coating, as we mentioned for Park et al.'s silicon nanotubes. Use of a carbon coating as a buffer is a common solution to the volume expansion, but this particular use of $\mathrm{SiO}_{\mathrm{x}}$ as a constraining layer has to do with its rigid feature. Some, if not most, of the carbon coatings can't fully contain the volume expansion, as shown in Figure 11(c), and would more than likely break up.

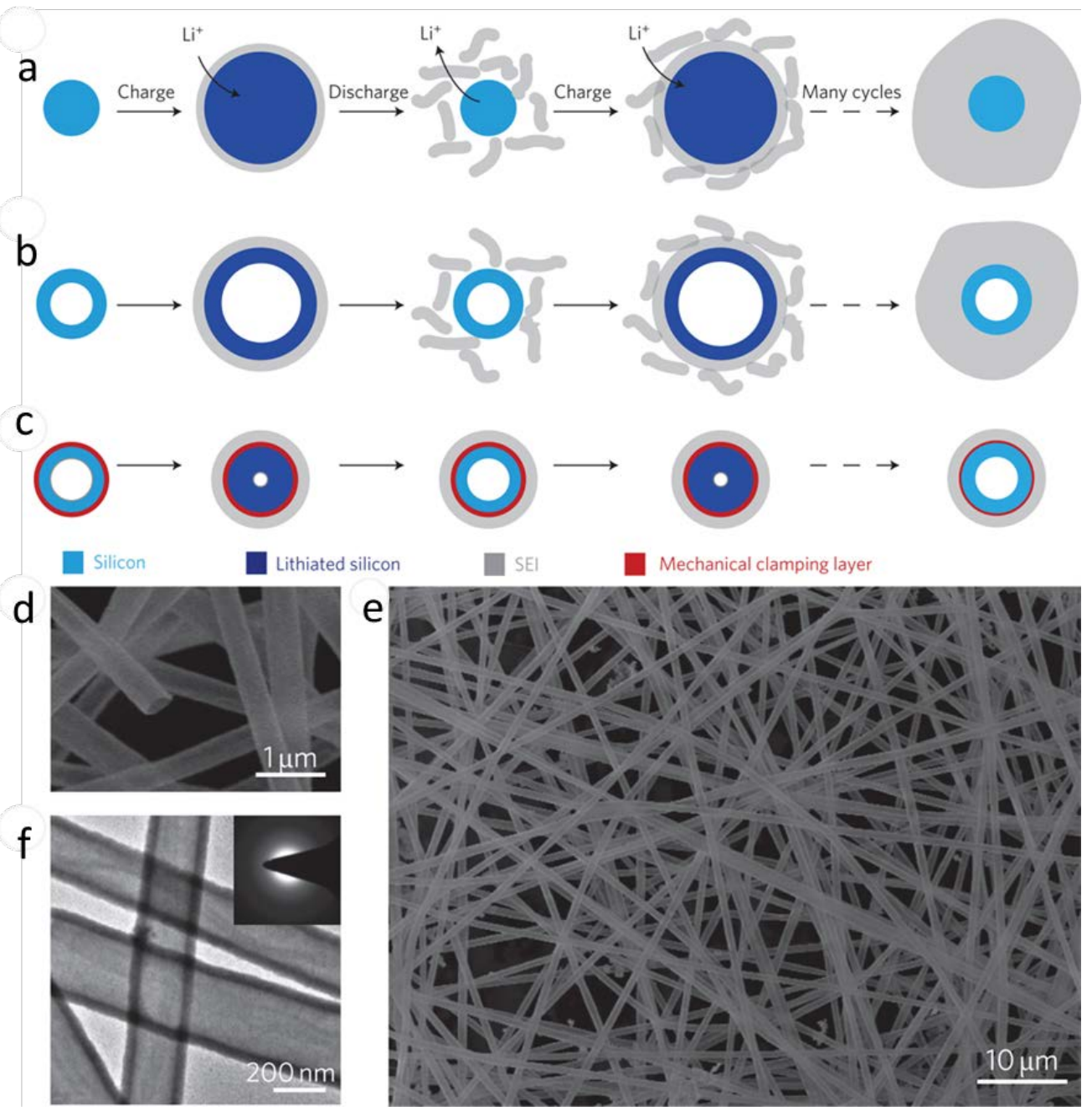

Figure 11: (a-c) Mechanisms displaying how the SEI layer is affected by the constant expansion through multiple charge and discharge phases for (a) a solid silicon nanowire, (b) silicon nanotube with no 
confining layer, and (c) silicon nanotube with a confining layer. (d,e) SEM images of synthesized DWSiNTs at high and low magnification, respectively. (f) TEM image of DWSiNTs, showing the uniform hollow structure with smooth tube walls. Reprinted with permission from Macmillan Publishers Ltd [Nature Nanotechnology] from Ref. [67]. Copyright 2012.

The double wall method could potentially only work so well with the nanotube structure because of its inward expansion. For a silicon nanowire, the silicon would have nowhere to expand except outwards, in which case the $\mathrm{SiO}_{\mathrm{x}}$ layer may not be able to withstand that stress. This brings out the merit of not only the confining layer but also the tube structure of the silicon. Figures 12(a,b) [67] show the performance of the DWSiNT anode over 6,000 cycles. Figure 12a shows that the capacity at the $12 \mathrm{C}$ rate has little to no fading after 6000 cycles. This figure indicates that the DWSiNT anode has very good stability for long term use and ought to be used in the future. It also shows good capacity retention from the first cycle. Once again, the capacity loss from the first cycle to the second is due to the SEI layer, which consumes some lithium ions. The finding that the capacity stabilized after the first cycle is evidence of a stable SEI film. Figure $12 \mathrm{~b}$ shows that the voltage profile remains constant throughout thousands of cycles, and Figures $12(\mathrm{c}, \mathrm{d})$ display how well the capacity is regained after increasing the current density and returning it to lower current density. 

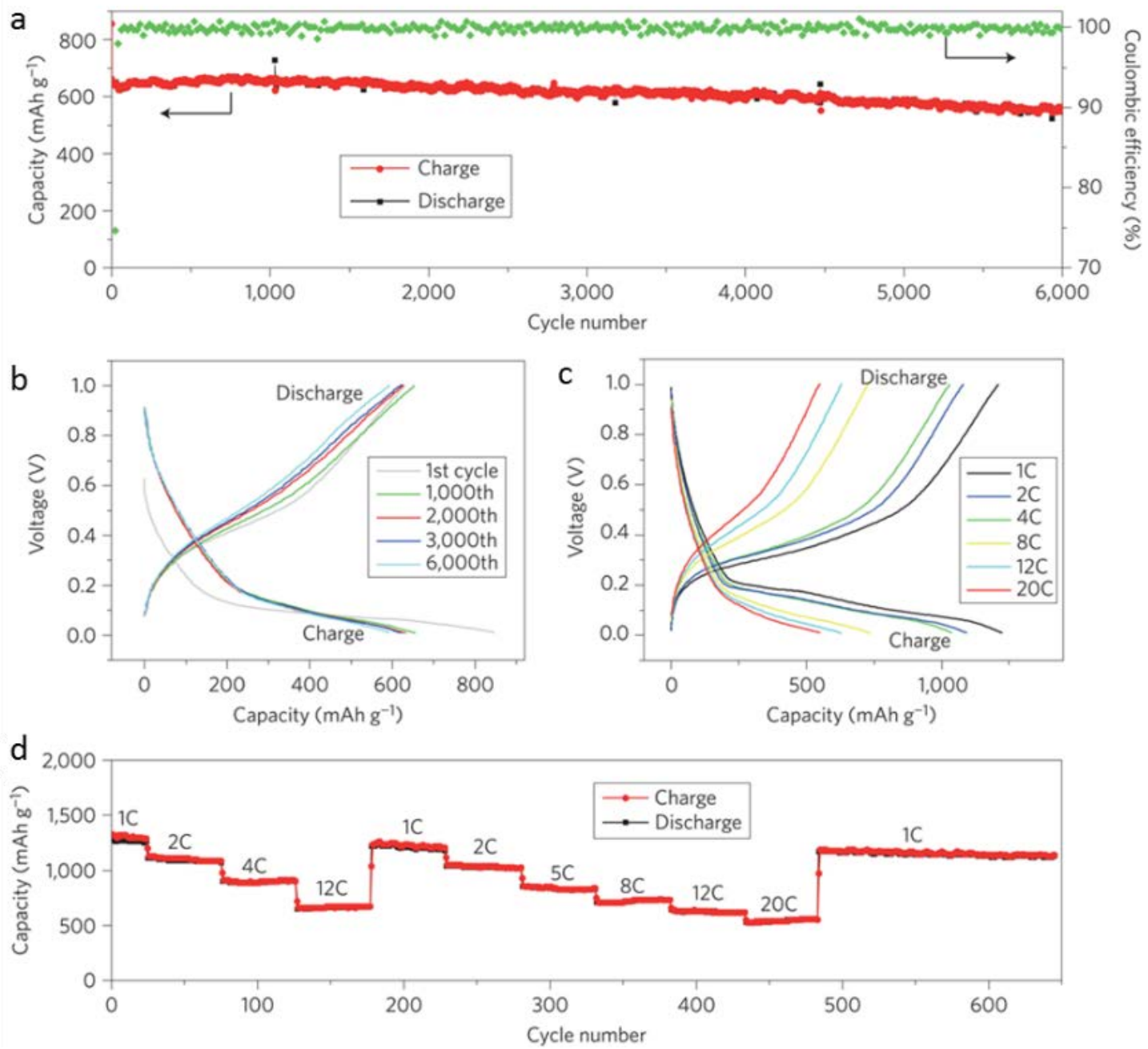

Figure 12: (a) Lithiation/delithiation capacity and coulombic efficiency of the DWSiNTs cycled at 12C for 6,000 cycles. (b) Voltage profiles plotted for the 1st, 1,000th, 2,000th, 3,000 ${ }^{\text {th }}$, and 6,000 th cycles. Galvanostatic charge/discharge profiles (c) and capacity retention (d) of DWSiNT anodes cycled at rates from 1C to 20C. Reprinted with permission from Macmillan Publishers Ltd [Nature Nanotechnology] from Ref. [67]. Copyright 2012.

\section{Silicon/Carbon Effectiveness}


Also under investigation for silicon-based batteries is the inclusion of novel carbon structures in a composite with silicon. Carbon is included because it can (1) work as a buffer for the volume expansion, (2) help increase and maintain electrical contact with surrounding conducting material, and (3) allow decent passage of lithium ions to flow to the silicon material. We will discuss a number of different carbon structures and carbon applications that have been developed in the past few years to improve silicon anode performance.

\subsection{Carbon Structures}

The structure of the carbon material used in the composite determines how it will interact with the silicon. For example, amorphous carbon has no crystalline structure and is very disordered. Acetylene black, which is a type of amorphous carbon, is commonly used as a conducting material. The purpose for most amorphous carbons is to establish and maintain electrical contact with the anode material. Because of its disorder, the amorphous structure also would not be good as a buffer for expansive anodes like silicon and tin. Amorphous carbon isn't strongly bonded compared to carbon materials like graphite or carbon nanotubes, so it is more likely to break apart and forced away by the expansion. Several studies have been completed using amorphous carbon as a coating for anode materials, especially for silicon [77-79]. Since amorphous material has no crystalline structure, there is no concern for too much damage during silicon expansion. However, it is difficult for such carbon to maintain a coating since the force of the expansion could cause it to break apart. Coating silicon particles with amorphous carbon has improved anode performance, but only to the extent of the improvement of electrical contact of the coating itself [77].

Another example of carbon structures is the carbon nanotube. The literature has many reports of studies using carbon nanotubes in lithium-ion batteries [15, 27, 80-84]. Carbon 
nanotubes have also been tested in nanoscale electronic devices, hydrogen storage materials, composites, and other electrochemical storage energy systems such as supercapacitors [27]. The tubular bond of the nanotubes is very strong, nearly 200 times that of steel. De las Casas and Li have proposed placing silicon within the tube to buffer the silicon expansion because of the bond strength. The carbon nanotube would stretch a bit, containing the expansion, and return to its original shape. This limiting of the volume expansion would result in better maintenance of the electrical contact with the surrounding conducting material [85]. Since the nanotube is carbon itself as well, it also contributes to maintaining electrical conductivity by its contact with the silicon particles and the surrounding conducting material.

Cui et al. [81] have proposed the use of carbon nanotubes as a free-standing electrode without the need for current collectors. In this arrangement, a carbon nanotube network is used throughout the silicon film, providing good conductivity and flexibility, as well as mechanical support, and acting as an embedded current collector. The composite film has a structure similar to that of steel-bar-reinforced concrete.

Yun et al. also applied this idea of reinforced concrete in a similar manner using graphene [86, 87]. Graphene, especially graphene oxide and reduced graphene oxides, has also been used for composites. Graphene essentially is a 2D carbon monolayer with a honeycomb lattice. Graphene is a very strong carbon structure and more than forty times stronger than diamond, but is also important to know that it is anisotropic in which the fracture occurs more quickly along the plane compared to perpendicular to the plane [88]. It has garnered much attention due to its unique physical and chemical properties such as amazingly high electrical conductivity, large theoretical surface area, exceptional mechanical flexibility, and superior chemical stability [89-91]. The graphene storage of one lithium ion on each side of graphene results in a $\mathrm{Li}_{2} \mathrm{C}_{6}$ stoichiometry that 
provides a specific capacity of $744 \mathrm{mAh} / \mathrm{g}$, which is twice that of graphite $(372 \mathrm{mAh} / \mathrm{g})[92,93]$. However, the formula of $\mathrm{Li}_{2} \mathrm{C}_{6}$ is only theoretical without computation and experimental evidence to prove the feasibility of this formula on pure graphene. The idea behind this has to do with a model of a "house of cards" formed by small graphene sheets where lithium are absorbed on the internal surfaces of nanopores [94]. Another graphene structure that has gained some recognition is reduced graphene oxide (RGO), i.e., graphene produced by reduction of graphene oxide (GO) by means of high-temperature heat treatment or use of reducing agents. The main difference with pure graphene is that RGO could still contain some oxide, depending on the reduction reaction. Graphene oxide is a promising precursor for synthesizing carbon-based nanostructures, because it can be synthesized in large quantities by oxidizing inexpensive graphite powders. The reduction of GO can be done by different methods to control the reduction conditions and thereby obtain desired properties [95-98]. Also, anode tests with RGO exhibited incredibly high-capacity values, $>2,000 \mathrm{mAh} / \mathrm{g}$, which is higher than the theoretical capacity of single-layer graphene, but unfortunately this capacity is lost after the formation of the SEI layer. This large capacity loss occurs because of the high specific surface area when the SEI forms on the surface of the active material. The much larger surface area leads to a higher capacity drop for graphene than graphite $[92,99]$. Due to the benefits from the graphene and RGO structure, these materials have been used quite often recently as an ideal carbon structure to mix with silicon to improve electrode performance. Most of the silicon/carbon composites reviewed in the next section use graphene and RGO.

\subsection{Carbon Implementation}

As previously demonstrated, the structure of the carbon material plays an important role in its performance as an anode. Among carbon materials studied, graphene has been used quite often 
in recent research. However, its benefit still greatly depends on how the material is applied. It is not enough to just mix silicon with any carbon structure and expect them to interact in an effective manner. Some methods such as carbon coating $[100,101]$ and mechanical mixing [102-104] (such as ball milling) have proven to have minimal effect and to be inefficient in preventing capacity fading. Other methods such as encapsulation [105-108], layering [109, 110], and binding [111] have seen better performance results because they do more than just mix silicon and carbon together. First, these methods result in improved electrical conductivity just by using carbon, more specifically, graphene in these cases. Second, they address the volume expansion, which also addresses the unstable SEI as that is a byproduct of the volume change. In this part, we only focus on the various methods to integrate graphene with silicon to fabricate composite anodes with improved capacity and cyclic stability.

\subsubsection{Encapsulation}

Encapsulation encases carbon in the silicon particle. This may not seem different from coating the particle with carbon, but researchers use encapsulation with empty spaces to allow room for silicon to expand $[107,108,112,113]$. Liu et al. implemented this empty space by developing a yolk-shell design. The silicon particle acts as the yolk and amorphous carbon as the shell, as displayed in Figure 13 [108]. The silicon particle is attached to a side of the carbon wall while the other is empty space. This attachment to the wall ensures that there is always contact with the carbon wall so that electrical conductivity is maintained. The carbon shell acts as an extension of the conductive network for the silicon particle, and if the contact with the silicon particle is lost, the contact with the rest of the conductive network is still retained. Figure $13 \mathrm{c}$ demonstrates the benefit to the void space by comparing encapsulated carbon to a conventional silicon carbon mixture and coating. The SEI reforming on top of fresh surface after the charging affects contact 
with the conducting material. With a carbon coating, the SEI will break apart at the expansive force of the silicon, which only creates an opening to fresh surface. Because the carbon coating alone can't stand that force, the empty space to make room for that expansion prevents the destruction of the shell. It is important to allow enough space for the silicon to expand, or it will still break apart regardless. With sufficient spacing, the SEI layer will be stable and have minimal capacity fading. 

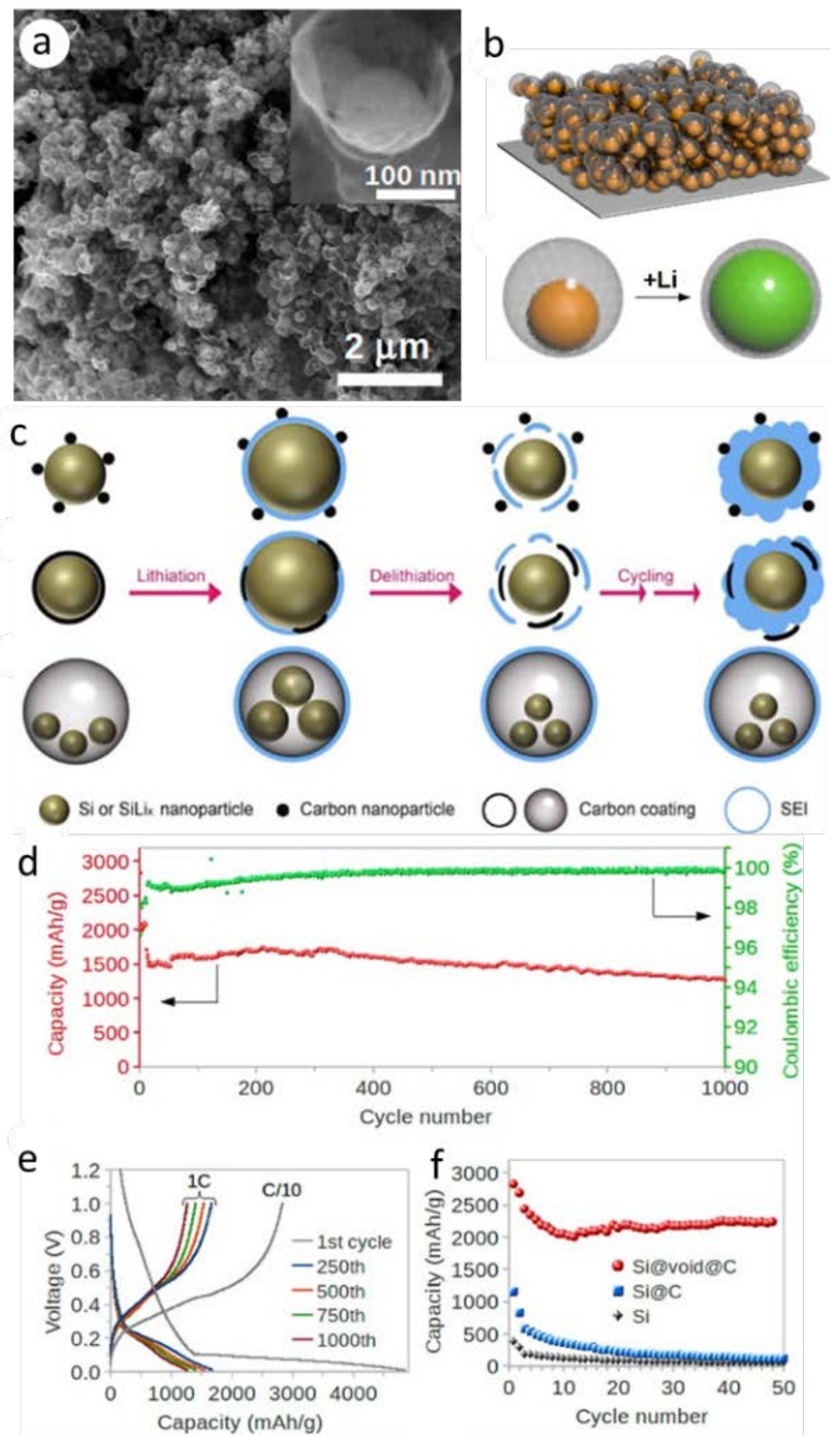

Figure 13: (c) Schematic illustrating several types of carbon-coated $\mathrm{Si}$ nanoparticles during $\mathrm{Li}^{+}$ insertion/extraction. Reprinted with permission from Ref. [107, 108]. American Chemical Society, 
Copyright 2012. (a) SEM images of synthesized void-encapsulated silicon powder. (b) Schematic of the yolk-shell design of the silicon encapsulation. (d-f) Electrochemical cycling results for void-encapsulated silicon electrodes. (d) Delithiation capacity and coulombic efficiency of the first 1000 galvanostatic cycles between 0.01 and $1 \mathrm{~V}$. The rate was $\mathrm{C} / 10$ for one cycle, then $\mathrm{C} / 3$ for 10 cycles, and $1 \mathrm{C}$ for the later cycles. (e) Charge and discharge voltage profiles. (f) Galvanostatic cycling of different silicon nanostructures. Reprinted with permission from Ref. [107, 108]. American Chemical Society, Copyright 2012.

Building upon the research of Liu et al., Luo et al. developed an encapsulation method that uses empty spaces. Instead of using a yolk-shell shape, they used the shape of crumpled paper. The crumpled graphene-encapsulated $\mathrm{Si}$ particles were made by a facile, capillary-driven assembly route in aerosol droplets, as seen in Figure 14a [107]. In this fabrication method, the RGO particles are wrapped around the silicon particles like paper. The folds and wrinkles of the graphene act as the empty space for the silicon to expand. In Figure 14d, the SEM image shows the folding morphologies of the crumpled graphene encapsulation and the wrapping of the majority of the silicon. The graphene shells are heavily crumpled with many folds and wrinkles because of capillary compression of the process. The crumpled graphene particles are stable and resistant against unfolding due to the large number of pi-pi stacked folds. They are also resistant to aggregation because the morphology of the crumpled graphene ball prevents the strong interparticle van der Waals attraction. 

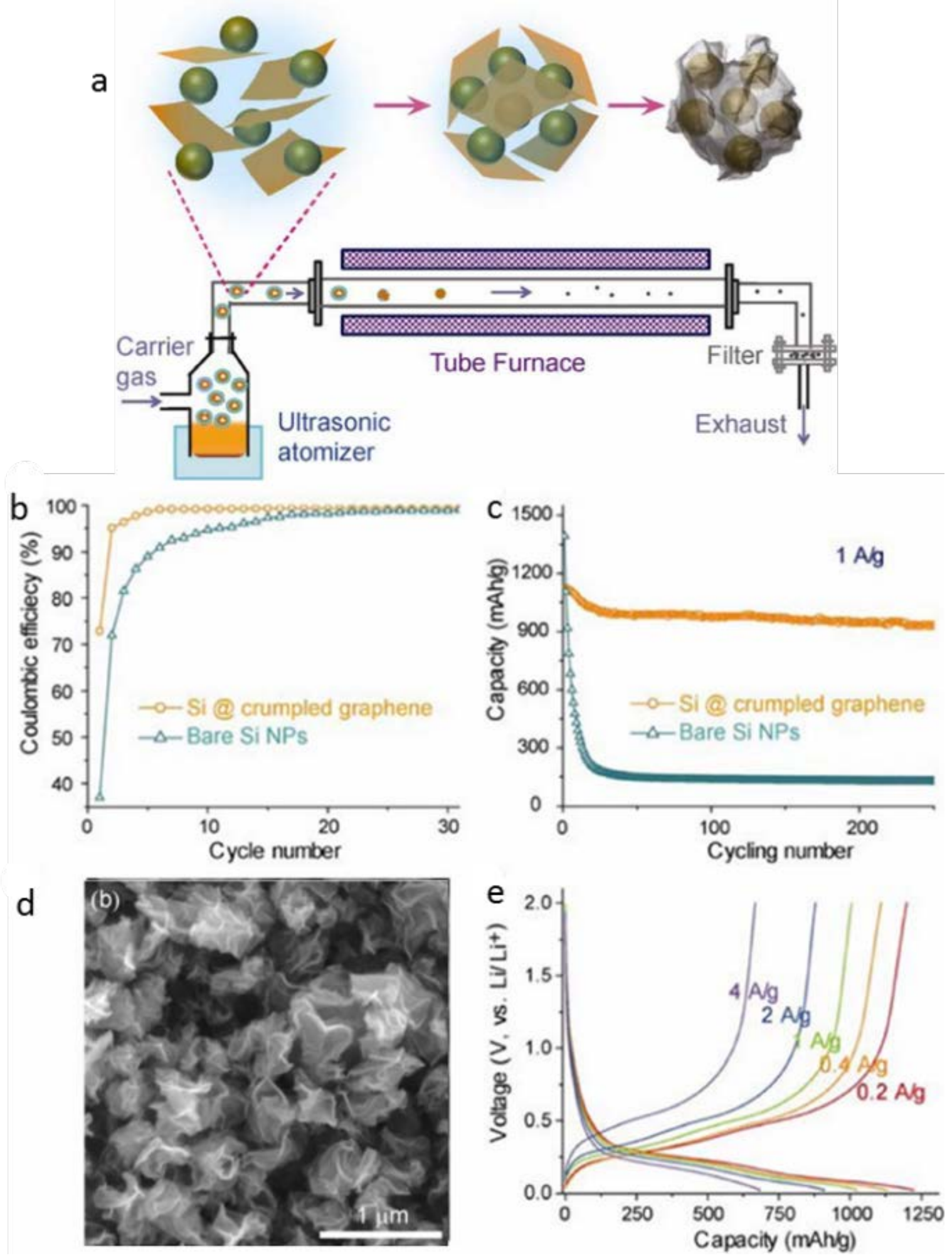

Figure 14: (a) Schematic drawings illustrating aerosol-assisted capillary assembly of crumpled-graphenewrapped Si nanoparticles. (b) Coulombic efficiency and (c) charge/discharge cycling test of the composite 
capsules in comparison to the unwrapped Si nanoparticles at a constant current density of $1 \mathrm{~A} / \mathrm{g}$. (d) SEM image of the Si nanoparticles encapsulated in the crumpled graphene. (e) Galvanostatic charge/discharge profiles of the composite capsule electrode at various current densities ranging from 0.2 to $4 \mathrm{~A} / \mathrm{g}$. Reprinted with permission from Ref. [107]. Copyright 2012, American Chemical Society.

\subsubsection{Layering}

In the layering method, RGO is deposited in layers with silicon nanoparticles in between each layer. Chang et al. had thought to tackle the silicon expansion by using RGO in layers, which would allow enough space for the silicon nanoparticle to expand. The multilayered Si/RGO anode contains units of silicon nanoparticles and continuous RGO films, as illustrated in Figure 15b [109]. Each isolated unit of silicon nanoparticles is connected by RGO films, which provide high electrical conductivity throughout the electrode. The RGO films closest to each silicon nanoparticle could easily deform to accommodate the volume change of silicon nanoparticles during the lithiation/delithiation process. The RGO films further away from each silicon nanoparticle could reconstruct to form 2-D networks of graphite due to van de Waals forces, providing mechanical stability. In Figure 15a, an SEM image shows a single layer of RGO on top of silicon nanoparticles, demonstrating how well the RGO film is layered on top of the silicon nanoparticles after being reduced from GO. On top of the conductivity and stability, the layer structure would also prevent silicon particles from breaking away from the electrode by having RGO as the top layer all the while allowing lithium ions to pass through easily. Chang et al. have also examined the use 3D porous nickel foam instead of the conventional copper foil current collector because it can improve mass loading of active materials, which would enhance the energy density. 

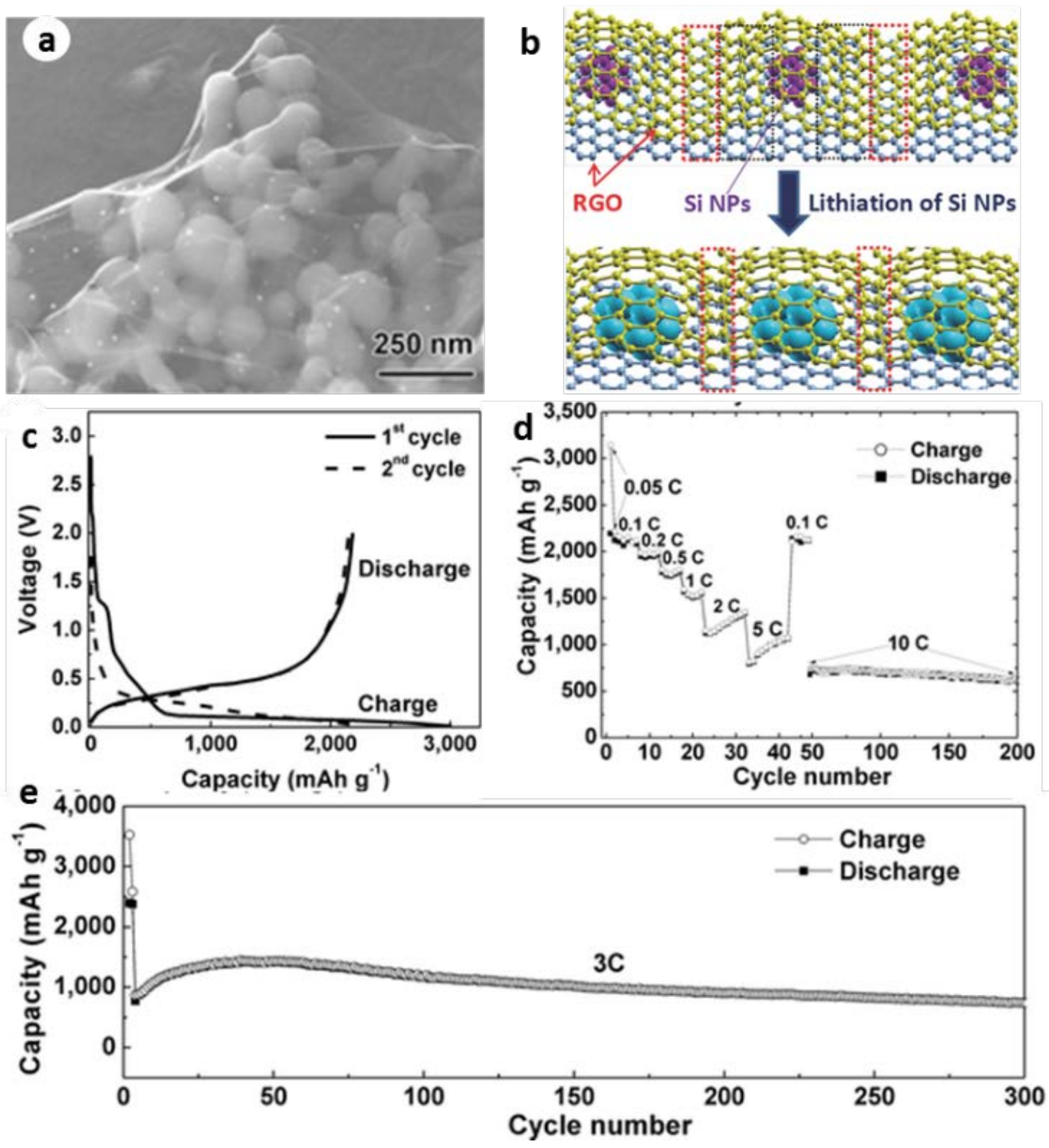

Figure 15: (a) SEM image of a layer of RGO covering a layer of Si nanoparticles. (b) Schematic representation of structural change of a multilayered $\mathrm{Si} / \mathrm{RGO}$ anode before and after lithiation. (c) Galvanostatic charge/discharge profiles of the first two cycles. (d) Charge/discharge capacities at various current rates as marked. (e) Cyclic performance of the multilayered $\mathrm{Si} / \mathrm{RGO}$ electrode cycled at $3 \mathrm{C}$ for 300 cycles. Reprinted with permission from Ref. [109]. Copyright 2014, Wiley-VCH. 


\subsubsection{Binding}

The final method involves binding silicon nanoparticles to the RGO structure. Chen et al. designed silicon/RGO nanocomposites via a simple in situ process with pre-synthesized GO and silicon dioxide and used a magnesiothermic reduction reaction. The process steps are shown in Figure 16 [111]. The GO, which was synthesized via the Hummers method, was coated with $\mathrm{SiO}_{2}$ via a hydrolysis reaction with tetraethyl orthosilicate (TEOS, $\mathrm{SiC}_{8} \mathrm{H}_{20} \mathrm{O}_{4}$ ) [114]. This reaction was followed by a magnesiothermic reduction by adding magnesium powder, then heating of the product to $650^{\circ} \mathrm{C}$ for $7 \mathrm{~h}$, and washing it with $\mathrm{HCl}$ and $\mathrm{HF}$ acid solution. The SEM and TEM images of the resulting product are shown in Figures 16a-d. In the TEM image, the silicon nanoparticles, indicated as dark spots, deposit on the RGO. Binding silicon nanoparticles in this way maintains contact between the silicon and the RGO. This improved contact allows for good electrical conductivity, even after the silicon expands. Figure 16e shows the cycling performance of a number of samples, including the bound silicon/RGO, physically mixed silicon/RGO, bulk silicon, and RGO. When comparing the physically mixed silicon/RGO and the bound silicon/RGO, the physical mix has higher capacity fading. This is most likely due to the detachment of silicon to the RGO. Without the binding procedure, silicon contact is easily lost with the RGO, thus reducing the conductivity. In addition, the ratio of silicon to carbon material is very important as the amount of silicon in a composite can greatly affect the performance. Figure 16f shows the cycling performance of anodes with different carbon ratios. The higher the silicon content, the higher the initial capacity, but also the higher the capacity fading. With higher silicon content, more silicon is added to the SEI, contributing to the expansion problem, and less carbon material is acting as a buffer to the silicon nanoparticles. For lower silicon content, more carbon is available to buffer the silicon nanoparticles, creating more 
stable performance, but little silicon is actually contributing to the overall capacity, lowering anode performance. Chen et al. found that the silicon/carbon optimal ratio was $40 \%$ carbon; $40 \%-60 \%$ tends to be around the usual ratio for most research to get the most benefit from both materials.
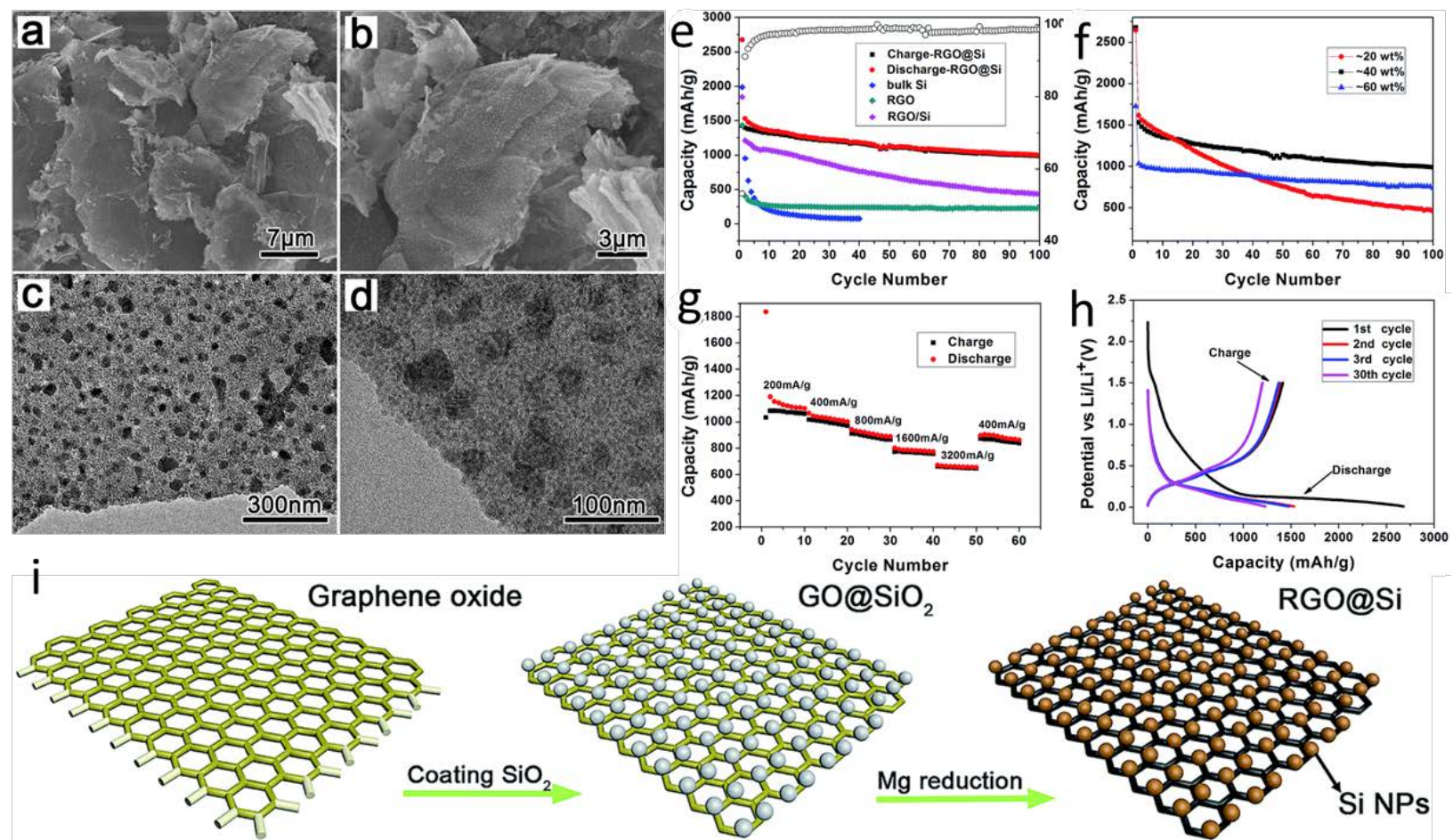

$\mathrm{GO} @ \mathrm{SiO}_{2}$

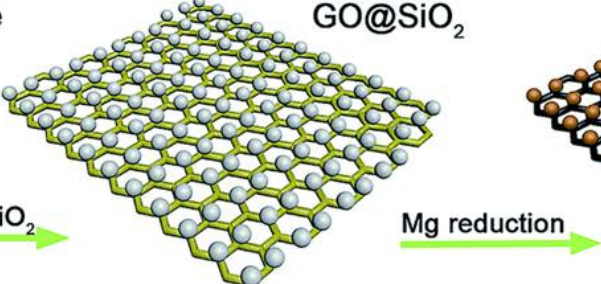

RGO@Si

Figure 16: Morphological characterizations of RGO@Si nanocomposites: (a) low- and (b) highmagnification SEM images, and (c) low- and (d) high-magnification TEM images. (e) Charge and discharge capacities and coulombic efficiency as a function of cycle numbers of the RGO@Si nanocomposites at a current density of $200 \mathrm{~mA} \mathrm{~g}^{-1}$ between 0.001 and $2.0 \mathrm{~V}$. (f) Cycling performance of RGO@Si nanocomposite electrodes with different amounts of carbon. (g) Rate performance of uniform RGO@Si nanocomposite electrode with a carbon content of $60 \%$. (h) Voltage profiles of the porous RGO@Si nanocomposites at $200 \mathrm{~mA} \mathrm{~g}^{-1}$. (i) Schematic illustrating the synthetic procedure for making firmly bonded RGO@Si nanocomposites. Reprinted with permission from Ref. [111]. Copyright 2015, Royal Society of Chemistry. 


\section{Conclusion and Perspective}

In summary, the main issue with silicon is the volume expansion that occurs during lithiation. As a result, the silicon nanoparticles suffer pulverization, create poor conductivity with the electrode, and cause an unstable SEI layer to form because of the fresh surfaces that are created after each cycle. After reviewing the literature on binders, silicon structures, and silicon/carbon composites, we reached a better understanding of approaches to these problems.

We found that binders are effective in maintaining electrical conductivity. Though there are numerous ways that can be achieved, binders are an effective and convenient means to improve electrode performance overall. Over the past years, little research has been done to improve the reversible capacity of silicon-based electrodes as rapid capacity fading is an issue. The binder is still a valuable means to stabilize performance, but improving the binder may not be the best path to silicon-based electrodes for next-generation lithium-ion batteries.

Research in changing the silicon structure has proven very fruitful. The silicon nanotube and nanowire have shown that they can address the main issue of pulverization that can't be resolved through normal silicon nanopowders. However, well-controlled nanostructures pose difficulties for the potential manufacturing procedure. The process by which some of these structures are synthesized could potentially result in high manufacturing cost, at least compared to traditional slurry methods. Large-scale manufacturing of anode materials needs to be technically feasible, low cost, and reproducible.

Ultimately, the use of silicon and novelty carbon structures seems to be the most effective means at addressing the problems involved with the silicon anode. This is evident from the many 
studies involving the use of novelty carbon, especially graphene and reduced graphene oxides. In addition to the fact that carbon materials help improve conductivity, the combination of carbon with silicon helps stabilize the SEI layer, contain the volume expansion, and buffer the effect of pulverization. Moreover, the synthesis process is potentially more cost effective and applicable to the convenient method of slurry formation.

As we stand, there are still some goals that need to be addressed for the silicon anode, in addition to the $1000 \mathrm{mAh} / \mathrm{g}$ capacity benchmark. Increased initial coulombic efficiency and higher capacity retention are two of the major goals. Reducing the lithium ions lost during the first cycle is still a challenge, depending on the fabrication approach, but just as there has been in the past few years, progress will be made in the near future.

\section{Acknowledgment}

G. Wu. acknowledges the financial support from the start-up funds of University at Buffalo along with National Science Foundation (CBET-1511528). This work was also partially supported by the U.S. Department of Energy under Contract DE-AC0206CH11357 from the Vehicle Technologies Office, Department of Energy, Office of Energy Efficiency and Renewable Energy (EERE).

\section{References}

[1] A. Yoshino, Angewandte Chemie International Edition, 51 (2012) 5798-5800.

[2] B. Scrosati, Electrochimica Acta, 45 (2000) 2461-2466.

[3] S. Megahed, W. Ebner, Journal of Power Sources, 54 (1995) 155-162. 
[4] A.N. Jansen, A.J. Kahaian, K.D. Kepler, P.A. Nelson, K. Amine, D.W. Dees, D.R. Vissers, M.M. Thackeray, Journal of Power Sources, 81-82 (1999) 902-905.

[5] L. Lu, X. Han, J. Li, J. Hua, M. Ouyang, Journal of Power Sources, 226 (2013) 272-288.

[6] B.A. Johnson, R.E. White, Journal of Power Sources, 70 (1998) 48-54.

[7] M.M. Thackeray, C. Wolverton, E.D. Isaacs, Energy \& Environmental Science, 5 (2012) 7854-7863.

[8] K. Smith, C.-Y. Wang, Journal of Power Sources, 160 (2006) 662-673.

[9] G. Lijun, S. Liu, R.A. Dougal, Components and Packaging Technologies, IEEE Transactions on, 25 (2002) 495-505.

[10] M. Contestabile, S. Panero, B. Scrosati, Journal of Power Sources, 92 (2001) 65-69.

[11] J. Shim, R. Kostecki, T. Richardson, X. Song, K.A. Striebel, Journal of Power Sources, 112 (2002) 222-230.

[12] W.-J. Zhang, Journal of Power Sources, 196 (2011) 13-24.

[13] M. Yoshio, H. Wang, K. Fukuda, T. Umeno, N. Dimov, Z. Ogumi, Journal of The Electrochemical Society, 149 (2002) A1598-A1603.

[14] M. Yoshio, H. Wang, K. Fukuda, Y. Hara, Y. Adachi, Journal of The Electrochemical Society, 147 (2000) 1245-1250.

[15] Y. Zhang, X.G. Zhang, H.L. Zhang, Z.G. Zhao, F. Li, C. Liu, H.M. Cheng, Electrochimica Acta, 51 (2006) 4994-5000.

[16] M. Inaba, Z. Siroma, Y. Kawatate, A. Funabiki, Z. Ogumi, Journal of Power Sources, 68 (1997) 221-226.

[17] C. Wang, H. Nakamura, H. Komatsu, M. Yoshio, H. Yoshitake, Journal of Power Sources, 74 (1998) 142-145. 
[18] Y. Zhu, M.D. Stoller, W. Cai, A. Velamakanni, R.D. Piner, D. Chen, R.S. Ruoff, ACS Nano, 4 (2010) 1227-1233.

[19] C. Erk, T. Brezesinski, H. Sommer, R. Schneider, J. Janek, ACS Applied Materials \& Interfaces, 5 (2013) 7299-7307.

[20] H. Li, X. Huang, L. Chen, G. Zhou, Z. Zhang, D. Yu, Y. Jun Mo, N. Pei, Solid State Ionics, 135 (2000) 181-191.

[21] J.R. Szczech, S. Jin, Energy \& Environmental Science, 4 (2011) 56-72.

[22] M.-H. Park, M.G. Kim, J. Joo, K. Kim, J. Kim, S. Ahn, Y. Cui, J. Cho, Nano Letters, 9 (2009) 3844-3847.

[23] U. Kasavajjula, C. Wang, A.J. Appleby, Journal of Power Sources, 163 (2007) 1003-1039.

[24] B.A. Boukamp, G.C. Lesh, R.A. Huggins, Journal of The Electrochemical Society, 128 (1981) 725-729.

[25] Y. He, X. Yu, Y. Wang, H. Li, X. Huang, Advanced Materials, 23 (2011) 4938-4941.

[26] J. Hassoun, K.-S. Lee, Y.-K. Sun, B. Scrosati, Journal of the American Chemical Society, 133 (2011) 3139-3143.

[27] W.X. Chen, J.Y. Lee, Z. Liu, Carbon, 41 (2003) 959-966.

[28] W.-M. Zhang, J.-S. Hu, Y.-G. Guo, S.-F. Zheng, L.-S. Zhong, W.-G. Song, L.-J. Wan, Advanced Materials, 20 (2008) 1160-1165.

[29] D.-H. Nam, K.-S. Hong, S.-J. Lim, M.-J. Kim, H.-S. Kwon, Small, 11 (2015) 2885-2892.

[30] H. Tian, F. Xin, X. Wang, W. He, W. Han, Journal of Materiomics, 1 (2015) 153-169.

[31] H. Wu, Y. Cui, Nano Today, 7 (2012) 414-429.

[32] V.A. Agubra, J.W. Fergus, Journal of Power Sources, 268 (2014) 153-162.

[33] P. Verma, P. Maire, P. Novák, Electrochimica Acta, 55 (2010) 6332-6341. 
[34] M. Nie, D. Chalasani, D.P. Abraham, Y. Chen, A. Bose, B.L. Lucht, The Journal of Physical Chemistry C, 117 (2013) 1257-1267.

[35] Y. Zhu, I.A. Shkrob, D.P. Abraham, Meeting Abstracts, MA2014-01 (2014) 30.

[36] S. Menkin, D. Golodnitsky, E. Peled, Electrochemistry Communications, 11 (2009) 17891791.

[37] V. Etacheri, O. Haik, Y. Goffer, G.A. Roberts, I.C. Stefan, R. Fasching, D. Aurbach, Langmuir, 28 (2012) 965-976.

[38] M. Nie, D.P. Abraham, Y. Chen, A. Bose, B.L. Lucht, The Journal of Physical Chemistry C, 117 (2013) 13403-13412.

[39] M.-H. Ryou, J. Kim, I. Lee, S. Kim, Y.K. Jeong, S. Hong, J.H. Ryu, T.-S. Kim, J.-K. Park, H. Lee, J.W. Choi, Advanced Materials, 25 (2013) 1571-1576.

[40] A. Magasinski, B. Zdyrko, I. Kovalenko, B. Hertzberg, R. Burtovyy, C.F. Huebner, T.F. Fuller, I. Luzinov, G. Yushin, ACS Applied Materials \& Interfaces, 2 (2010) 3004-3010. [41] J. Li, R.B. Lewis, J.R. Dahn, Electrochemical and Solid-State Letters, 10 (2007) A17-A20. [42] Z. Chen, L. Christensen, J.R. Dahn, Electrochemistry Communications, 5 (2003) 919-923. [43] J. Li, L. Christensen, M.N. Obrovac, K.C. Hewitt, J.R. Dahn, Journal of The Electrochemical Society, 155 (2008) A234-A238.

[44] S. Komaba, K. Shimomura, N. Yabuuchi, T. Ozeki, H. Yui, K. Konno, The Journal of Physical Chemistry C, 115 (2011) 13487-13495.

[45] J.-S. Bridel, T. Azaïs, M. Morcrette, J.-M. Tarascon, D. Larcher, Journal of The Electrochemical Society, 158 (2011) A750-A759.

[46] J.S. Bridel, T. Azaïs, M. Morcrette, J.M. Tarascon, D. Larcher, Chemistry of Materials, 22 (2010) 1229-1241. 
[47] N.S. Hochgatterer, M.R. Schweiger, S. Koller, P.R. Raimann, T. Wöhrle, C. Wurm, M. Winter, Electrochemical and Solid-State Letters, 11 (2008) A76-A80.

[48] W.-R. Liu, M.-H. Yang, H.-C. Wu, S.M. Chiao, N.-L. Wu, Electrochemical and Solid-State Letters, 8 (2005) A100-A103.

[49] H. Buqa, M. Holzapfel, F. Krumeich, C. Veit, P. Novák, Journal of Power Sources, 161 (2006) 617-622.

[50] G. Liu, S. Xun, N. Vukmirovic, X. Song, P. Olalde-Velasco, H. Zheng, V.S. Battaglia, L. Wang, W. Yang, Advanced Materials, 23 (2011) 4679-4683.

[51] L. Shen, Q. Che, H. Li, X. Zhang, Advanced Functional Materials, 24 (2014) 2630-2637.

[52] C. Ban, Z. Wu, D.T. Gillaspie, L. Chen, Y. Yan, J.L. Blackburn, A.C. Dillon, Advanced Materials, 22 (2010) E145-E149.

[53] X. Zhu, P. Zhang, S. Xu, X. Yan, Q. Xue, ACS Applied Materials \& Interfaces, 6 (2014) $11665-11674$.

[54] I. Kovalenko, B. Zdyrko, A. Magasinski, B. Hertzberg, Z. Milicev, R. Burtovyy, I. Luzinov, G. Yushin, Science, (2011).

[55] Y. Yao, M.T. McDowell, I. Ryu, H. Wu, N. Liu, L. Hu, W.D. Nix, Y. Cui, Nano Letters, 11 (2011) 2949-2954.

[56] H. Kim, B. Han, J. Choo, J. Cho, Angewandte Chemie, 120 (2008) 10305-10308.

[57] H. Jia, P. Gao, J. Yang, J. Wang, Y. Nuli, Z. Yang, Advanced Energy Materials, 1 (2011) 1036-1039.

[58] X. Li, M. Gu, S. Hu, R. Kennard, P. Yan, X. Chen, C. Wang, M.J. Sailor, J.-G. Zhang, J. Liu, Nat Commun, 5 (2014). 
[59] J.P. Maranchi, A.F. Hepp, P.N. Kumta, Electrochemical and Solid-State Letters, 6 (2003) A198-A201.

[60] S. Bourderau, T. Brousse, D.M. Schleich, Journal of Power Sources, 81-82 (1999) 233-236.

[61] X. Chen, X. Li, F. Ding, W. Xu, J. Xiao, Y. Cao, P. Meduri, J. Liu, G.L. Graff, J.-G. Zhang, Nano Letters, 12 (2012) 4124-4130.

[62] A.M. Chockla, J.T. Harris, V.A. Akhavan, T.D. Bogart, V.C. Holmberg, C. Steinhagen, C.B. Mullins, K.J. Stevenson, B.A. Korgel, Journal of the American Chemical Society, 133 (2011) 20914-20921.

[63] C.K. Chan, R.N. Patel, M.J. O’Connell, B.A. Korgel, Y. Cui, ACS Nano, 4 (2010) 14431450.

[64] R. Ruffo, S.S. Hong, C.K. Chan, R.A. Huggins, Y. Cui, The Journal of Physical Chemistry C, 113 (2009) 11390-11398.

[65] C.K. Chan, R. Ruffo, S.S. Hong, Y. Cui, Journal of Power Sources, 189 (2009) 1132-1140.

[66] C.K. Chan, H. Peng, G. Liu, K. Mcllwrath, X.F. Zhang, R.A. Huggins, Y. Cui, Nat Nano, 3 (2008) 31-35.

[67] H. Wu, G. Chan, J.W. Choi, I. Ryu, Y. Yao, M.T. McDowell, S.W. Lee, A. Jackson, Y. Yang, L. Hu, Y. Cui, Nat Nano, 7 (2012) 310-315.

[68] S. Goriparti, E. Miele, F. De Angelis, E. Di Fabrizio, R. Proietti Zaccaria, C. Capiglia, Journal of Power Sources, 257 (2014) 421-443.

[69] J. Cho, Journal of Materials Chemistry, 20 (2010) 4009-4014.

[70] M.R. Zamfir, H.T. Nguyen, E. Moyen, Y.H. Lee, D. Pribat, Journal of Materials Chemistry A, 1 (2013) 9566-9586. 
[71] X. Su, Q. Wu, J. Li, X. Xiao, A. Lott, W. Lu, B.W. Sheldon, J. Wu, Advanced Energy Materials, 4 (2014) n/a-n/a.

[72] J. Xiao, W. Xu, D. Wang, D. Choi, W. Wang, X. Li, G.L. Graff, J. Liu, J.-G. Zhang, Journal of The Electrochemical Society, 157 (2010) A1047-A1051.

[73] X.H. Liu, L. Zhong, S. Huang, S.X. Mao, T. Zhu, J.Y. Huang, ACS Nano, 6 (2012) 15221531.

[74] M. Gu, Y. Li, X. Li, S. Hu, X. Zhang, W. Xu, S. Thevuthasan, D.R. Baer, J.-G. Zhang, J. Liu, C. Wang, ACS Nano, 6 (2012) 8439-8447.

[75] M.T. McDowell, I. Ryu, S.W. Lee, C. Wang, W.D. Nix, Y. Cui, Advanced Materials, 24 (2012) 6034-6041.

[76] R. Teki, M.K. Datta, R. Krishnan, T.C. Parker, T.-M. Lu, P.N. Kumta, N. Koratkar, Small, 5 (2009) 2236-2242.

[77] S.H. Ng, J. Wang, D. Wexler, S.Y. Chew, H.K. Liu, The Journal of Physical Chemistry C, 111 (2007) 11131-11138.

[78] M. Noh, Y. Kwon, H. Lee, J. Cho, Y. Kim, M.G. Kim, Chemistry of Materials, 17 (2005) 1926-1929.

[79] M.M. Rahman, J.-Z. Wang, M.F. Hassan, D. Wexler, H.K. Liu, Advanced Energy Materials, 1 (2011) 212-220.

[80] S.H. Ng, J. Wang, Z.P. Guo, J. Chen, G.X. Wang, H.K. Liu, Electrochimica Acta, 51 (2005) 23-28.

[81] L.-F. Cui, L. Hu, J.W. Choi, Y. Cui, ACS Nano, 4 (2010) 3671-3678.

[82] W. Wang, P.N. Kumta, ACS Nano, 4 (2010) 2233-2241.

[83] W. Wang, R. Epur, P.N. Kumta, Electrochemistry Communications, 13 (2011) 429-432. 
[84] X.-M. Liu, Z.d. Huang, S.w. Oh, B. Zhang, P.-C. Ma, M.M.F. Yuen, J.-K. Kim, Composites Science and Technology, 72 (2012) 121-144.

[85] C. de las Casas, W. Li, Journal of Power Sources, 208 (2012) 74-85.

[86] Q. Yun, X. Qin, W. Lv, Y.-B. He, B. Li, F. Kang, Q.-H. Yang, Carbon, 93 (2015) 59-67.

[87] J.-Z. Wang, C. Zhong, S.-L. Chou, H.-K. Liu, Electrochemistry Communications, 12 (2010) 1467-1470.

[88] Z. Ni, H. Bu, M. Zou, H. Yi, K. Bi, Y. Chen, Physica B: Condensed Matter, 405 (2010) $1301-1306$.

[89] E. Yoo, J. Kim, E. Hosono, H.-s. Zhou, T. Kudo, I. Honma, Nano Letters, 8 (2008) $2277-$ 2282.

[90] J.K. Lee, K.B. Smith, C.M. Hayner, H.H. Kung, Chemical Communications, 46 (2010) $2025-2027$.

[91] P. Lian, X. Zhu, S. Liang, Z. Li, W. Yang, H. Wang, Electrochimica Acta, 55 (2010) 39093914.

[92] R. Raccichini, A. Varzi, S. Passerini, B. Scrosati, Nat Mater, 14 (2015) 271-279.

[93] M. Liang, L. Zhi, Journal of Materials Chemistry, 19 (2009) 5871-5878.

[94] Y. Liu, J.S. Xue, T. Zheng, J.R. Dahn, Carbon, 34 (1996) 193-200.

[95] Y. Zhu, S. Murali, W. Cai, X. Li, J.W. Suk, J.R. Potts, R.S. Ruoff, Advanced Materials, 22 (2010) 3906-3924.

[96] M. Zhou, Y. Wang, Y. Zhai, J. Zhai, W. Ren, F. Wang, S. Dong, Chemistry - A European Journal, 15 (2009) 6116-6120.

[97] J. Zhu, T. Zhu, X. Zhou, Y. Zhang, X.W. Lou, X. Chen, H. Zhang, H.H. Hng, Q. Yan, Nanoscale, 3 (2011) 1084-1089. 
[98] H.-C. Tao, L.-Z. Fan, Y. Mei, X. Qu, Electrochemistry Communications, 13 (2011) 13321335.

[99] Z.-S. Wu, W. Ren, L. Xu, F. Li, H.-M. Cheng, ACS Nano, 5 (2011) 5463-5471.

[100] N. Dimov, S. Kugino, M. Yoshio, Electrochimica Acta, 48 (2003) 1579-1587.

[101] H.S. Kim, K.Y. Chung, B.W. Cho, Journal of Power Sources, 189 (2009) 108-113.

[102] S.-L. Chou, J.-Z. Wang, M. Choucair, H.-K. Liu, J.A. Stride, S.-X. Dou, Electrochemistry Communications, 12 (2010) 303-306.

[103] H. Xiang, K. Zhang, G. Ji, J.Y. Lee, C. Zou, X. Chen, J. Wu, Carbon, 49 (2011) 1787-1796.

[104] R. Hu, W. Sun, Y. Chen, M. Zeng, M. Zhu, Journal of Materials Chemistry A, 2 (2014) 9118-9125.

[105] C. He, S. Wu, N. Zhao, C. Shi, E. Liu, J. Li, ACS Nano, 7 (2013) 4459-4469.

[106] X. Zhou, Y.-X. Yin, L.-J. Wan, Y.-G. Guo, Advanced Energy Materials, 2 (2012) 10861090.

[107] J. Luo, X. Zhao, J. Wu, H.D. Jang, H.H. Kung, J. Huang, The Journal of Physical Chemistry Letters, 3 (2012) 1824-1829.

[108] N. Liu, H. Wu, M.T. McDowell, Y. Yao, C. Wang, Y. Cui, Nano Letters, 12 (2012) 33153321.

[109] J. Chang, X. Huang, G. Zhou, S. Cui, P.B. Hallac, J. Jiang, P.T. Hurley, J. Chen, Advanced Materials, 26 (2014) 758-764.

[110] X. Zhou, Y.-X. Yin, L.-J. Wan, Y.-G. Guo, Chemical Communications, 48 (2012) 21982200.

[111] Y. Chen, N. Du, H. Zhang, D. Yang, RSC Advances, 5 (2015) 46173-46180.

[112] H. Wu, G. Zheng, N. Liu, T.J. Carney, Y. Yang, Y. Cui, Nano Letters, 12 (2012) 904-909. 
[113] X. Li, P. Meduri, X. Chen, W. Qi, M.H. Engelhard, W. Xu, F. Ding, J. Xiao, W. Wang, C. Wang, J.-G. Zhang, J. Liu, Journal of Materials Chemistry, 22 (2012) 11014-11017.

[114] G. De, B. Karmakar, D. Ganguli, Journal of Materials Chemistry, 10 (2000) 2289-2293. 
$\underline{\text { Author photos and short bios }}$

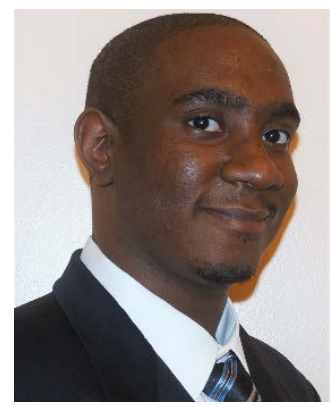

Anix Casimir was a Master graduate students in Department of Chemical and Biological engineering at University at Buffalo, SUNY. He received his Bachelor's at Rochester Institute of Technology in 2014 . His research focused on silicon and silicon/ carbon composite anode materials for Lithium-Ion Batteries.

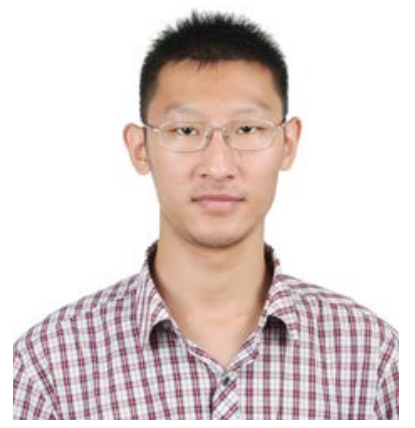

Hanguang Zhang is a Ph.D. student at University at Buffalo, SUNY. He obtained his Bachelor and Master degrees from Sichuan University (2013) and UB (2015), respectively. His research project is to synthesize three-dimensional porous carbon materials for energy conversion and storage.

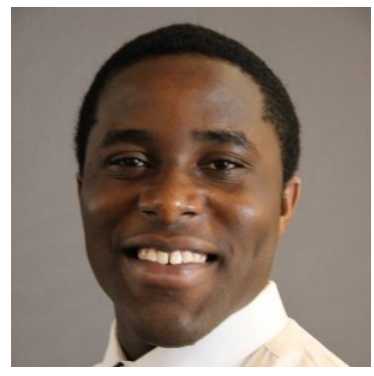

Ogechi Ogoke is a PhD student at the University at Buffalo, SUNY. He completed his bachelor's degree at the University of Maine in chemical engineering. His current research focuses on high performance nanostructured materials for Li-ion and beyond Li-ion batteries. 


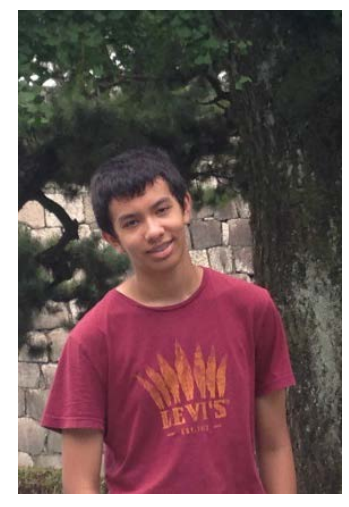

Joseph C. Amine is a senior student at Hinsdale Central High School in Illinois. He had summer internship at Argonne National Laboratory (3 times) in Illinois, Hanyang University in Seoul of South Korea, Stanford University in California and Peking University in Shenzhen of China.

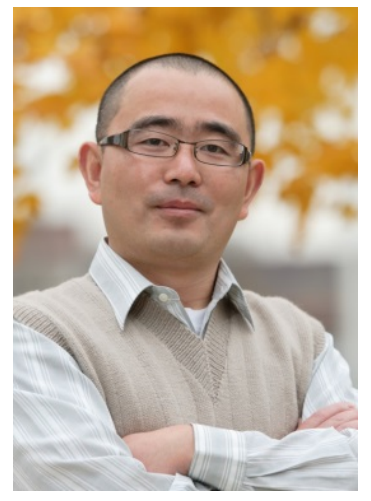

Jun Lu is a chemist at Argonne National Laboratory. His research interests focus on the electrochemical energy storage and conversion technology, with main focus on beyond Li-ion battery technology. Dr. Lu earned his bachelor degree in Chemistry Physics from University of Science and Technology of China (USTC) in 2000. He completed his Ph.D. from the Department of Metallurgical Engineering at University of Utah in 2009 with a major research on metal hydrides for reversible hydrogen storage application. He is the awardee of the first DOE-EERE postdoctoral fellow under Vehicles Technology Program from 2011-201 3. Dr. Lu has authored/co-authored more than 150 peer-reviewed research articles, including Nature; Chem. Rev.;Nature Commun.; JACS; etc, and has filed over 20 patents and patent applications 


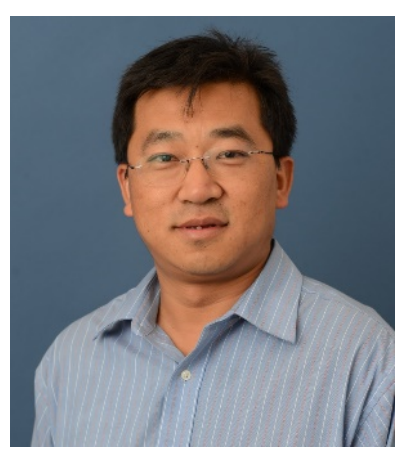

Gang $W u$ is an Assistant Professor in the Department of Chemical and Biological Engineering at the University at Buffalo (UB), SUNY since August 2014. Prior to joining $U B$, he was a staff scientist at Los Alamos National Laboratory (LANL). He completed his Ph.D. studies at the Harbin Institute of Technology in 2004 followed by extensive postdoctoral trainings at Tsinghua University (2004-2006), the University of South Carolina (2006-2008), and LANL (2008-201 0). His research focuses on functional materials and catalysts for electrochemical energy storage and conversion. 